\title{
A Multi-Commodity Flow Formulation for the Optimal Design of Wastewater Treatment Networks th
}

\author{
Xin Cheng, Xiang Li* \\ Department of Chemical Engineering, Queen's University, 19 Division Street, Kingston, ON, K'L 3N6, \\ Canada
}

\begin{abstract}
The wastewater treatment network (WWTN) optimization problem can be viewed as an extension of the generalized pooling problem (GPP), where not only the different streams are mixed and split, but also some chemical components are removed from the system. It is well known that a strong formulation is key to efficient global solution of GPP, and multicommodity flow formulations have been recognized as such strong formulations. The paper presents the first multi-commodity flow formulation for the WWTN optimization problem. We discuss the challenges in adapting the classical multi-commodity flow approaches for a WWTN, and propose the conditions under which the selected commodities are adequate for modeling a WWTN. We prove that the proposed formulation is at least as strong as a classical component based formulation. We show the significant computational advantage of the proposed formulation over the component based formulation through 16 problem instances from the literature.
\end{abstract}

Keywords: Wastewater treatment, Multi-commodity flow, Global optimization

\section{Introduction}

Wastewater treatment systems are essential for protecting public health and the environment. Despite of their rapid development in the past decades, current capacity of wastewater

\footnotetext{
¿ (C)2019. This manuscript version is made available under the CC-BY-NC-ND 4.0 license http://creativecommons.org/licenses/by-nc-nd/4.0/. The final publication is available at elsevier via https://doi.org/10.1016/j.compchemeng.2019.106681

${ }^{*}$ Corresponding author

Email addresses: x.cheng@queensu.ca (Xin Cheng), xiang.li@queensu.ca (Xiang Li)
} 
treatment system remains lagging behind. It is estimated that on average $70 \%$ of the generated wastewater is treated among high-income countries, followed by $38 \%, 28 \%$ and $8 \%$ among upper-middle-income, lower-middle-income and low-income countries [1]. Urgently, more wastewater treatment plants need to be built or upgraded to handle the increasing wastewater generation caused by the ongoing industrialization and urbanization.

To raise the efficiency of a wastewater treatment plant, it is not only important to adopt the state-of-the-art treatment technologies and a robust process control system, but also a design that guarantees the optimal pathway of each waste stream. The optimal design of wastewater treatment network (WWTN) is a critical step to accomplish this task. It seeks to: i), select the best treatment technologies that are to be included in the plant; ii) for the selected treatment technologies, determine the optimal allocation of water streams. A study by Forshomi et al. (2017) [2] shows that, an optimal design of effluent treatment system can reduce the total cost by up to $19.5 \%$ and the electricity cost by up to $12 \%$, for a stream assisted gravity drainage oil sand operation. In the context of industrial water usage, considering both the WWTN and the water using network in optimization can further reduce the cost $[3,4,5,6]$. Moreover, Karuppiah and Grossmann [7] considered the uncertainty in the contaminant generation and removal for the integrated water network and solved a multiscenario problem.

Methods that solve the WWTN problem roughly fall into two categories: insight-based methods and optimization-based methods. During the early stage of the water network studies, most researchers considered the insight-based methods. Wang and Smith [8] showed that a distributed format of the network can greatly reduce the amount of wastewater to be treated for single-contaminant cases, using water pinch analysis. Kuo and Smith [9] extended Wang and Smith's work to multi-contaminant cases, and they also proposed to optimize the network design over a superstructure that contains all connections under consideration. However, due to the complexity of the network, they only consider part of the possible connections among the units with water pinch analysis, leading to a possibly suboptimal solution. Although insight-based methods cannot guarantee an optimal design, they have been successful for single-contaminant cases because of convenience for application 
[10]. The optimization-based methods find an optimal design by solving a mathematical programming problem formulated upon a predefined superstructure. Galan and Grossmann [11] proposed to cast the WWTN problem as a mathematical programming problem, with the assumption that the process has no water loss and the blending obeys linear blending rules. The behavior of the treatment units is described with constant removal ratios of the contaminants. While the model developed by Galan and Grossmann [11] has been widely accepted for optimization based research on WWTN, there exist other optimization models that consider different problem settings. For example, Huang et al. [12] considered water loss in the model; Song et al. [13] considered variable removal ratios calculated from the Monod and Andrews plug-flow reactor model for biological treatment units. More detailed review of water network design methods can be found in [14].

While optimization-based methods have attracted growing interests, they bring computational challenges. The problem is often cast as a nonlinear program (NLP), or a mixedinteger nonlinear program (MINLP) with the integer variables indicating whether certain treatment technology is selected. A MINLP formulation is also obtained in case active connections in the network are subject to minimum flow rates. The nonlinearity in the model comes from the bilinear terms that describe the blending and splitting effects of the waste streams. Due to the nonconvexity, a local solver often reports a local optimum, and a global solver requires considerable computational effort to find a global optimum. Many efforts have been made to speed up the solution procedure of a WWTN problem. Early works did not consider global optimality guarantee. For example, in $[15,16]$, the authors solved a sequence of linear programs (LP) to obtain a good starting point and solve the problem with a local NLP solver. Similarly, in $[17,18]$, the authors used a sequential approach that first constructs a LP (by using insight-based knowledge or fixing the split fraction variable) and then solves a NLP. Meta-heuristic methods such as genetic algorithms [19] and adaptive random search methods [20] have also been considered. Over the last two decades, more attention has been paid to global optimization based methods. Zamora and Grossmann [21] solved the optimization problem to global optimality using a branch and contract algorithm for a wastewater treatment system including 3 waste streams and 3 treatment units. However, 
the solution time was over $1000 \mathrm{CPU}$ seconds for such a small problem. Later on, Karuppiah and Grossmann [6] developed a new spatial branch and contract algorithm for improved solution efficiency, and applied the algorithm to an integrated water system. Karuppiah and Grossmann [22] also developed a multiscenario optimization model to address the uncertainties in the problem, and developed a Lagrangian decomposition based spatial branch and cut algorithm for solving the resulting multiscenario MINLP problem. Bergamini et al. [23] developed a piecewise outer-approximation algorithm and demonstrated its effectiveness through two WWTN problems. The Multi-parametric disaggregation technique (MDT) [24] has also been adopted to solve the WWTN global optimization problem $[25,26]$, where some continuous variables are discretized such that the optimization problem can be represented as a mixed-integer linear program (MILP). While the prior global optimization research on WWTN reported efficient application to simple WWTN problems, the global optimization methods may converge very slowly for problems with more complicated structures.

It is widely recognized that the global optimization procedure can be accelerated by not only more efficient solution methods, but also stronger problem formulations. A typical example in which strong formulations have significant computational advantages is the pooling problem. Since first studied by Haverly [27], the pooling problem and its extension, the generalized pooling problem (GPP), have been formulated in different ways in order for better solution efficiency. Recently, multi-commodity flow formulations [28] [29] have attracted more attention for GPP because they are proved to be stronger than the classical p-formulation and shown to render significant reduction in solution time. As a matter of fact, the WWTN problem can be treated as an extension of the GPP. These two problems both try to find an optimal stream allocation over a network while satisfying the quality constraints at the terminals. However, in the WWTN problem chemical components (i.e., contaminants) in the streams can be removed from the network, but in the GPP they cannot. Therefore, applying the multi-commodity flow formulations to the WWTN requires further investigation.

The main contribution of this paper is the development of a multi-commodity flow formulation for the WWTN problems, and the commodity flow selection criteria that are needed 
for the validity of the formulation. The rest of the paper is organized as follows: Section 2 gives problem description and a classical WWTN formulation in the literature. Section 3 discusses the concept of commodity flow, explains the challenges of modeling a WWTN with commodity flows, and proves the sufficient and necessary conditions under which a WWTN can be modeled with commodity flows. In addition, single-contaminant commodity flows are shown to always satisfy the conditions. Section 4 gives the multi-commodity flow formulation, and proves that it is at least as strong as the classical formulation presented in Section 2. Section 5 demonstrates the computational advantages of the proposed multicommodity formulation through case study problems in the literature. Section 6 summarizes the conclusions and discusses potential future work.

\section{Problem Statement}

\subsection{Problem Description}

The WWTN optimization problem considered in this paper assumes a steady-state process without water loss and linear blending rules. Any actual treatment unit is modeled as a linear filter that has different constant removal ratios for different contaminants. Since total mass of contaminants is very low in comparison to the total mass of water in a waste stream, the mass loss due to removal of contaminants is neglected.

A superstructure of WWTN is shown in Fig 1. It contains sets of splitters (inlet/outlet splitters), mixers (inlet/outlet mixers) and treatment units. For simplicity, each treatment unit in the superstructure represents one or multiple units with the same treatment technology and the same contaminant removal ratios; the optimization only determines the total capacity of the same type of units, but not how the total capacity is realized by a number of actually units. Wastewater streams entering each inlet splitter are assumed to have known constant flowrates and concentrations of contaminants. A wastewater stream can either go to any of the inlet mixers, or go directly to the outlet mixer without treatment. The output of each inlet mixer is sent to a corresponding treatment unit. The output of a treatment unit can either be sent back to any of the inlet splitters, or be sent to the outlet mixer for discharge. 


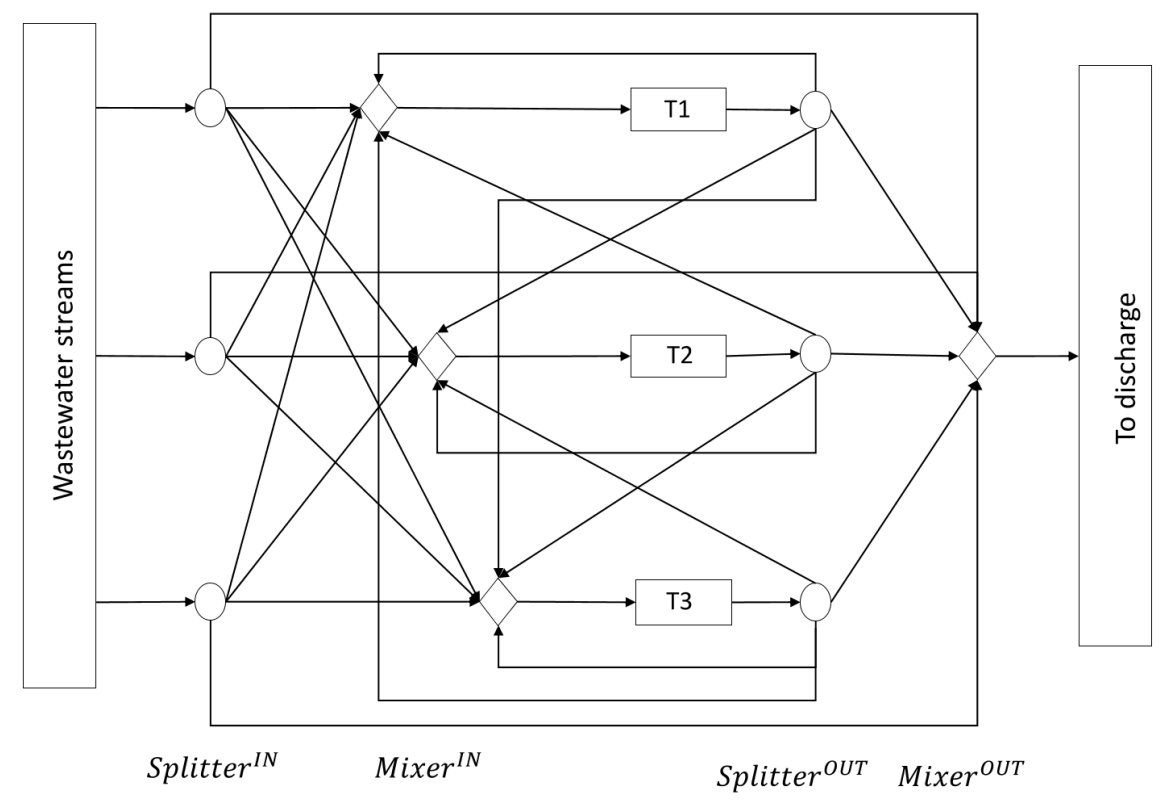

Figure 1: The superstructure of a wastewater treatment network with three units

\subsection{The Contaminant Flow Formulation}

There exist two WWTN formulations in the literature, a concentration based formulation [30] and a contaminant flow formulation [11]. Since the contaminant flow formulation often provides a better computational performance as shown by Castro et al. [31], we give this formulation below and later compare it with our proposed multi-commodity flow formulation. The contaminant flow formulation has been presented in different forms in the literature, which are essentially the same except some minor differences (such as variable bounds). The formulation given below (called C formulation) is the same as the one in [31], and the equivalence of the two formulations is explained in the appendix. Table 1 lists the symbols used in the formulation. Note that unit 'ppm' is part per million in terms of mass, so unit $10^{3} \mathrm{ppm}$ is equivalent to $\mathrm{kg} / \mathrm{t}$. 


\begin{tabular}{ll}
\hline Indices and Sets & \\
$s \in S$ & All splitters \\
$s i \in S_{I} \subset S$ & Inlet splitter \\
$s o \in S_{O} \subset S$ & Outlet splitter \\
$m \in M$ & All mixers \\
$m i \in M_{I} \subset M$ & Inlet mixers \\
$m o \in M_{O} \subset M$ & Outlet mixers \\
$t \in T$ & Treatment units \\
$c \in C$ & Contaminants \\
$v \in V$ & Commodities \\
$(t, s o) \in A_{O}$ & Set of allowable connection between so and $t$ \\
$(m i, t) \in A_{I}$ & Set of allowable connection between $m i$ and $t$ \\
& \\
Parameters & \\
$\beta_{s i}^{I N}(\mathrm{t} / \mathrm{h})$ & Total flow going through inlet splitter $s i$ \\
$\alpha_{c, s i}^{I N}(\mathrm{~kg} / \mathrm{h})$ & Contaminant flow $c$ going through inlet splitter $s i$ \\
$\theta_{c}^{O U T}\left(10^{3} \mathrm{ppm}\right)$ & Upper limit of discharge concentration of contaminant $c$ \\
$\theta_{t, c}^{I N}\left(10^{3} \mathrm{ppm}\right)$ & Upper limit of concentration of $c$ entering treatment unit $t$ \\
$\gamma_{t, c}$ & Removal ratio of $c$ in treatment units $t$ \\
$R$ & Contaminant conversion matrix \\
$R^{\prime}$ & Commodity conversion matrix \\
$r_{t, v, v}^{\prime}$ & Entries in $R^{\prime}$ for treatment unit $t$ \\
$\lambda_{c, v}\left(10^{3} \mathrm{ppm}\right)$ & Pre-defined concentration of $c$ in commodity $v$ \\
$\eta_{t}(\mathrm{t} / \mathrm{h})$ & Processing capacity of treatment unit $t$ \\
& \\
Variables & \\
$F_{t}^{T}(\mathrm{t} / \mathrm{h})$ & Total flow going through treatment unit $t$ \\
$F_{s, m}(\mathrm{t} / \mathrm{h})$ & Total flow from splitter $s$ to mixer $m$ \\
$f_{s i, v}(\mathrm{t} / \mathrm{h})$ & Commodity flow $v$ going through inlet splitter $s i$ \\
$f_{s, m, c}(\mathrm{~kg} / \mathrm{h})$ & Contaminant flow from splitter $s$ to mixer $m$ \\
$f_{m i, t, c}^{i}(\mathrm{~kg} / \mathrm{h})$ & Contaminant flow entering treatment unit $t$ \\
$f_{t, s o, c}^{o}(\mathrm{~kg} / \mathrm{h})$ & Contaminant flow leaving treatment unit $t$ \\
$f_{s, m, v}(\mathrm{t} / \mathrm{h})$ & Commodity flow from splitter $s$ to mixer $m$ \\
$f_{m i, t, v}^{i}(\mathrm{t} / \mathrm{h})$ & Commodity flow entering treatment unit $t$ \\
$f_{t, s o, v}^{o}(\mathrm{t} / \mathrm{h})$ & Commodity flow leaving treatment unit $t$ \\
$x_{s, m}$ & Split fraction variable at splitter s \\
\hline & \\
& \\
& \\
&
\end{tabular}

Table 1: The list of symbols used in the WWTN formulations 


\section{The C Formulation}

$$
\begin{aligned}
& \min \sum_{t \in T} F_{t}^{T} \\
& \text { s.t. } \beta_{s i}^{I N} x_{s i, m}=F_{s i, m} \\
& \alpha_{c, s i}^{I N} x_{s i, m}=f_{s i, m, c} \\
& f_{t, s o, c}^{o} x_{s o, m}=f_{s o, m, c} \\
& F_{t}^{T} x_{s o, m}=F_{s o, m} \\
& \sum_{m \in M} x_{s, m}=1 \\
& f_{m i, t, c}^{i}=\sum_{s \in S} f_{s, m i, c} \\
& F_{t}^{T}=\sum_{s \in S} F_{s, m i} \\
& f_{t, s o, c}^{o}=\left(1-\gamma_{t, c}\right) f_{m i, t, c}^{i} \\
& F_{t}^{T}=\sum_{m \in M} F_{s o, m} \\
& f_{t, s o, c}^{o}=\sum_{m \in M} f_{s o, m, c} \\
& \sum_{s \in S} f_{s, m i, c} \leq F_{t}^{T} \theta_{t, c}^{I N} \\
& \sum_{s \in S} f_{s, m o, c} \leq \sum_{s i \in S_{I}} \beta_{s i}^{I N} \theta_{c}^{O U T} \\
& 0 \leq f_{t, s o, c}^{o} \leq\left(1-\gamma_{t, c}\right) \sum_{s i \in S_{I}} \alpha_{s i, c}^{I N} \\
& \forall s i \in S_{I}, m \in M \\
& \forall s i \in S_{I}, m \in M, c \in C \\
& \forall(t, s o) \in A_{O}, m \in M, c \in C, \\
& \forall(t, s o) \in A_{O}, m \in M \\
& \forall s \in S \\
& \forall(m i, t) \in A_{I}, c \in C \\
& \forall(m i, t) \in A_{I} \\
& \forall(m i, t) \in A_{I},(t, s o) \in A_{O}, c \in C \\
& \forall(t, s o) \in A_{O} \\
& \forall(t, s o) \in A_{O}, c \in C \\
& \forall(m i, t) \in A_{I}, c \in C \\
& \forall c \in C, m o \in M_{O} \\
& \forall(t, s o) \in A_{O}, c \in C \\
& 0 \leq F_{t}^{T} \leq \eta_{t} \\
& \forall t \in T \\
& 0 \leq x_{s, m} \leq 1
\end{aligned}
$$

Objective. Since the operational cost is largely dependent on the flowrates entering the treatment units, the objective is to minimize the total flow entering treatment units. If the investment cost of the WWTN also needs to be considered, the formulation will need 
to include integer variables, but it won't influence the applicability of our proposed multicommodity formulation.

Mass Balances at Splitters. Eq. (C-2) and Eq. (C-5) represent total mass balances at inlet and outlet splitters, respectively. $\beta_{s i}^{I N}$ is the constant total flow entering splitter $s i$, and $F_{t}^{T}$ is the total flow entering treatment unit $t . F_{s, m}$ is total flow going from splitter $s$ to mixer $m$. Set $A_{O}$ defines which outlet splitter is connected to which treatment unit. Split fraction variable $x_{s, m}$ represents the fraction of flow to mixer $m$ in the total inflows to splitter $s$. Eq. (C-6) states that outputs of the splitters must all go to mixers. Similarly, Eq. (C-3) and Eq. (C-4) represent contaminant mass balances at splitters, where $\alpha_{s i, c}^{I N}$ represents the constant inflow rate of contaminant $c$ to splitter si and $f_{t, s o, c}^{o}$ represents the flow rate of contaminant $c$ to splitter so. We require that all flows be mass flows for convenience of establishing mass balance equations. Eq. (C-4) and Eq. (C-5) include bilinear terms and the are the sources of nonconvexity.

Mass Balances at Mixers. Eq. (C-7) and Eq. (C-8) represent the contaminant balances and total mass balances at inlet mixers, respectively. Set $A_{I}$ defines which inlet mixer is connected with which treatment unit.

Mass Balances at Treatment Units. Eq. (C-9) represents the contaminant balances at treatment units, i.e., the flow rate of contaminant $c$ after the treatment in unit $t$ is $1-r_{r, c}$ of the flow rate of contaminant $c$ before the treatment.

Strengthening Constraints. Eq. (C-10) and Eq. (C-11) are obtained via reformulation linearization technique [32], specifically, by summing Eq. (C-5) and Eq. (C-4) over $m \in M$, respectively. These two constraints are redundant for defining the decision making problem but help to accelerate the global optimization procedure.

Quality Constraints. Eq. (C-12) and Eq. (C-13) represent the upper limits of the contaminants at the treatment units and the outlet mixer. Note that the quality constraints on the inflows of treatment units are due to practical considerations, such as to prevent equipment corrosion or sensor failure. 
Variable Bounds. Eq. (C-14)-(C-16) define the bounds of the variables in the bilinear terms. The upper bound in Eq. (C-14) is estimated from the extreme case in which all waste streams enter treatment unit $t$. The upper bound in Eq. (C-15) comes from the processing capacity limit of the treatment unit.

\section{Modeling the WWTN Problem with Commodity Flows}

\subsection{Commodity Flow Representation of a Stream}

A stream in a chemical engineering process contains water and one or multiple chemical components. Naturally, the compositions of streams in a process network can be modeled via balance equation on each individual component; in this case, a stream is viewed as mixture of a water flow and a set of component flows each containing only one chemical component. The component flow representation of streams is widely accepted in process systems engineering; for WWTN, it already appeared in the literature in 1990s [30], [11], where component flows are called contaminant flows because all chemical components of interest are contaminants.

The commodity flow representation of a stream was first proposed by Alfaki and Haugland [28] in the context of GPP. In their work, a stream is viewed as mixture of commodity flows rather than component flows, and each commodity flow is defined to be the flow that enters a source of the pooling network. The difference between the commodity flow representation and component flow representation of a stream is visualized in Figure 2. Alfaki and Haugland proved that their multi-commodity flow formulation is stronger than (rigorously speaking, at least as strong as) the classical p-formulation (which can be viewed as a special component flow based formulation), in the sense that it leads to tighter convex relaxations and therefore results in more efficient global optimization. Later, Boland et al. [29] extended the concept of commodity flow and allowed a commodity flow to be a flow entering a source, a flow entering a terminal, or part of a flow that originally comes from a particular source and eventually goes to a particular terminal. Note that when the commodity flow is

a flow from a source, the percentages of the components in the flow are known; when the 


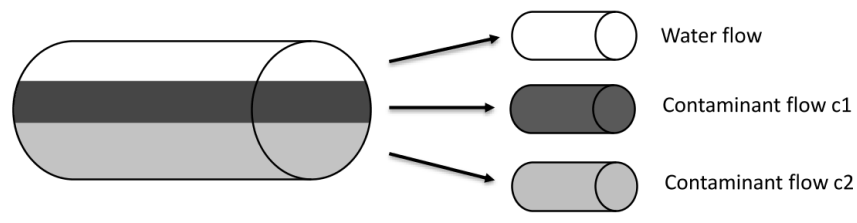

(a) Contaminant flow representation

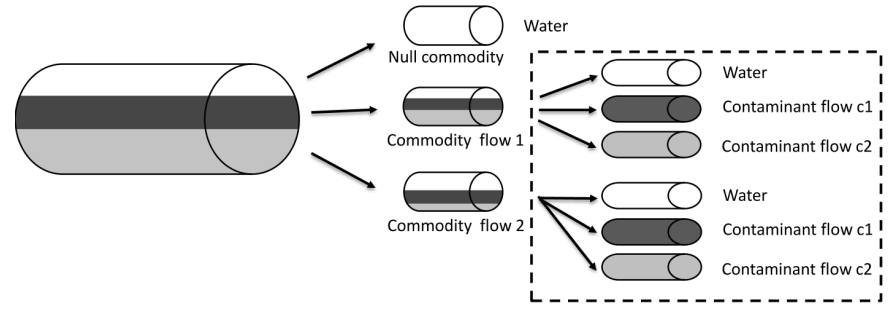

(b) Commodity flow representation

Figure 2: Comparison of contaminant (component) flow representation and commodity flow representation of a stream

commodity flow is a flow entering a terminal, the percentages of the components in the flow are variables and dependent on the optimal solution.

In this paper, we generalize the concept of commodity and allow a commodity to be any mixture of water and the components, in order to develop a multi-commodity flow formulation that is applicable to WWTN. In the subsequent subsection, we will see why such generalization is needed for WWTN. Let the contaminants of interest be $c_{1}, c_{2}, \cdots, c_{m}$, and the percentages of the contaminants in a commodity $v_{i}$ are $\lambda_{c_{1}, v_{i}}, \lambda_{c_{2}, v_{i}}, \cdots, \lambda_{c_{n}, v_{i}}$, respectively. Suppose we select $n$ commodities, $v_{1}, \cdots, v_{n}$, to model all streams in the problem. The compositions of the commodities can be summarized in the following commodity composition matrix:

$$
\boldsymbol{\Lambda}=\left[\begin{array}{ccc}
\lambda_{c_{1}, v_{1}} & \cdots & \lambda_{c_{1}, v_{n}} \\
\vdots & \ddots & \vdots \\
\lambda_{c_{m}, v_{1}} & \cdots & \lambda_{c_{m}, v_{n}}
\end{array}\right]
$$

In Alfaki and Haugland's work [28], $\boldsymbol{\Lambda}$ contains the compositions of flows entering the sources, which are known constants. In the work of Boland et al.[29], $\boldsymbol{\Lambda}$ can also be the compositions of flows entering the terminals, which are variables in the optimization. In this paper, we allow $\boldsymbol{\Lambda}$ to have any values that, together with water, enable a valid and efficient 


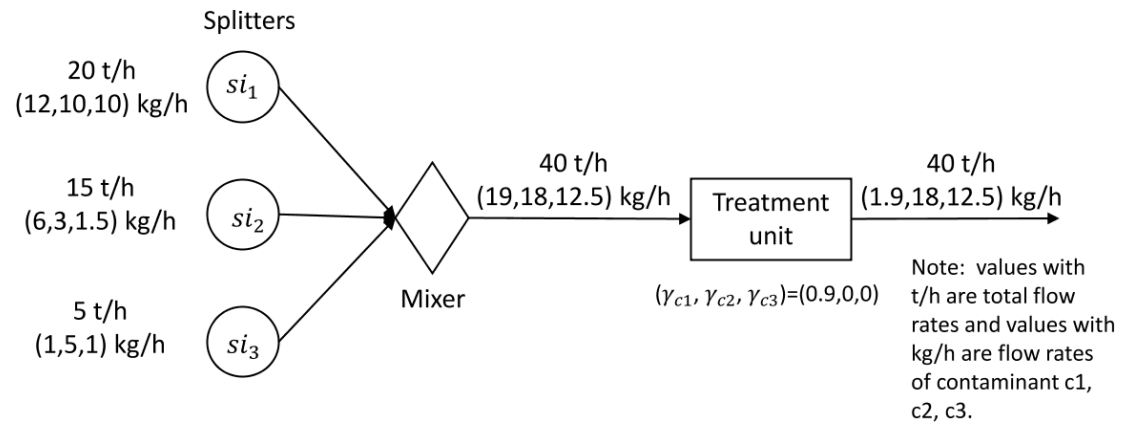

Figure 3: An example for contaminant flow representation

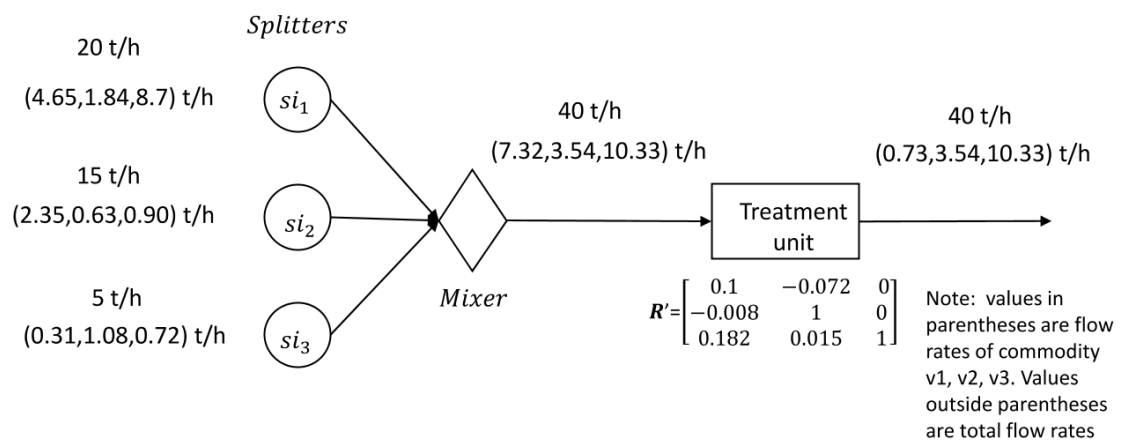

Figure 4: An example for commodity flow representation

multi-commodity flow formulation for WWTN. Next, we discuss the conditions under which the selected commodities are adequate for representing all streams in the network.

\subsection{A Simple Example for Contaminant and Commodity Flow Representations}

Fig. 3 shows a simple WWTN that contains 3 inlet waste streams and 1 treatment unit. There are three contaminants $\left(x_{1}, c_{2}, c_{3}\right)$, and their individual flow rates are shown in the parentheses with unit of $\mathrm{kg} / \mathrm{h}$, while the total flow rates are shown with unit of $\mathrm{t} / \mathrm{h}$. The removal ratios for $x_{1}, c_{2}, c_{3}$ at the treatment unit are $0.9,0,0$, respectively.

To represent all streams using commodity flows, the following commodity composition 
matrix is selected

$$
\boldsymbol{\Lambda}=\left[\begin{array}{ccc}
\lambda_{c_{1}, v_{1}} & \cdots & \lambda_{c_{1}, v_{3}} \\
\vdots & \ddots & \vdots \\
\lambda_{c_{3}, v_{1}} & \cdots & \lambda_{c_{3}, v_{3}}
\end{array}\right]=\left[\begin{array}{ccc}
2.5 & 0.2 & 0 \\
0 & 4.5 & 0.2 \\
0.2 & 0.2 & 1.0
\end{array}\right] \quad\left(10^{3} \mathrm{ppm}\right)
$$

which means that we use the following three commodities to represent the streams:

- $v_{1}$ contains $2.5 \times 10^{3} p p m c_{1}, 0.2 \times 10^{3} p p m c_{3}$ and no $c_{2}$,

- $v_{2}$ contains $0.2 \times 10^{3} p p m c_{1}, 4.5 \times 10^{3} p p m c_{2}$, and $0.2 \times 10^{3} p p m c_{3}$,

- $v_{3}$ contains $0.2 \times 10^{3}$ ppm $c_{2}, 1.0 \times 10^{3} \mathrm{ppm} c_{3}$, and no $c_{1}$.

The waste stream entering splitter $s i_{1}$ can now be viewed as the mixture of $4.65 \mathrm{t} / \mathrm{h}$ of $v_{1}$, $1.84 \mathrm{t} / \mathrm{h}$ of $v_{2}, 8.7 \mathrm{t} / \mathrm{h} v_{3}$ and a pure water flow (such that the total flow is $20 \mathrm{t} / \mathrm{h}$ ). The rates of contaminants entering $s i_{1}$ are:

$$
\boldsymbol{\Lambda}\left[\begin{array}{c}
4.65 \\
1.84 \\
8.7
\end{array}\right]=\left[\begin{array}{ccc}
2.5 & 0.2 & 0 \\
0 & 4.5 & 0.2 \\
0.2 & 0.2 & 1.0
\end{array}\right]\left[\begin{array}{c}
4.65 \\
1.84 \\
8.7
\end{array}\right]=\left[\begin{array}{c}
12 \\
10 \\
10
\end{array}\right](\mathrm{kg} / \mathrm{h})
$$

Commodity flow representation for other streams can be found in Fig. 4. The commodity conversion matrix $R^{\prime}$ of the treatment unit converts the inlet commodity flows into the outlet commodity flows. Here is how this matrix is generated. Let the inlet commodity flows be $f_{v_{1}}^{i, v}, f_{v_{2}}^{i, v}, f_{v_{3}}^{i, v}$ and the outlet commodity flows be $f_{v_{1}}^{o, v}, f_{v_{2}}^{o, v}, f_{v_{3}}^{o, v}$. Then the inlet contaminant flows and the outlet contaminant flows can be represented as

$$
\boldsymbol{\Lambda}\left[\begin{array}{c}
f_{v_{1}}^{i, v} \\
f_{v_{2}}^{i, v} \\
f_{v_{3}}^{i, v}
\end{array}\right], \quad \boldsymbol{\Lambda}\left[\begin{array}{c}
f_{v_{1}}^{o, v} \\
f_{v_{2}}^{o, v} \\
f_{v_{3}}^{o, v}
\end{array}\right]
$$


respectively. According to the removal ratios of the treatment unit,

$$
\left[\begin{array}{ccc}
1-0.9 & 0 & 0 \\
0 & 1-0 & 0 \\
0 & 0 & 1-0
\end{array}\right] \boldsymbol{\Lambda}\left[\begin{array}{l}
f_{v_{1}}^{i, v} \\
f_{v_{2}}^{i, v} \\
f_{v_{3}}^{i, v}
\end{array}\right]=\boldsymbol{\Lambda}\left[\begin{array}{l}
f_{v_{1}}^{o, v} \\
f_{v_{2}}^{o, v} \\
f_{v_{3}}^{o, v}
\end{array}\right]
$$

so

$$
\left[\begin{array}{l}
f_{v_{1}}^{o, v} \\
f_{v_{2}}^{o, v} \\
f_{v_{3}}^{o, v}
\end{array}\right]=\boldsymbol{\Lambda}^{-1}\left[\begin{array}{ccc}
0.1 & 0 & 0 \\
0 & 1 & 0 \\
0 & 0 & 1
\end{array}\right] \boldsymbol{\Lambda}\left[\begin{array}{l}
f_{v_{1}}^{i, v} \\
f_{v_{2}}^{i, v} \\
f_{v_{3}}^{i, v}
\end{array}\right]=\left[\begin{array}{ccc}
0.1 & -0.072 & 0 \\
-0.008 & 1 & 0 \\
0.182 & 0.015 & 1
\end{array}\right]\left[\begin{array}{l}
f_{v_{1}}^{i, v} \\
f_{v_{2}}^{i, v} \\
f_{v_{3}}^{i, v}
\end{array}\right]=R^{\prime}\left[\begin{array}{l}
f_{v_{1}}^{i, v} \\
f_{v_{2}}^{i, v} \\
f_{v_{3}}^{i, v}
\end{array}\right]
$$

Note that we can also choose a different set of commodity flows to represent the same streams, i.e., the choice of $\boldsymbol{\Lambda}$ is not unique. On the other hand, an arbitrary set of commodities may not be able to represent given streams. In the next subsection, we discuss commodity selection criteria under which there exists a $\boldsymbol{\Lambda}$ that is suitable for modeling a given WWTN.

\subsection{Commodity Selection Criteria for WWTN}

First, the selected commodities should be able to represent all incoming waste streams. Let the incoming waste streams be $s_{1}, \cdots, s_{w}$, their total flows be

$$
\boldsymbol{\beta}^{I N}=\left(\beta_{s i_{1}}^{I N}, \cdots, \beta_{s i_{w}}^{I N}\right)
$$

and the contaminant flows in the waste streams be

$$
\boldsymbol{\alpha}^{I N}=\left[\begin{array}{ccc}
\alpha_{c_{1}, s i_{1}}^{I N} & \cdots & \alpha_{c_{1}, s i_{w}}^{I N} \\
\vdots & \ddots & \vdots \\
\alpha_{c_{m}, s i_{1}}^{I N} & \cdots & \alpha_{c_{m}, s i_{w}}^{I N}
\end{array}\right]
$$

then the following proposition states the conditions under which the incoming waste streams can be represented by the commodities. 


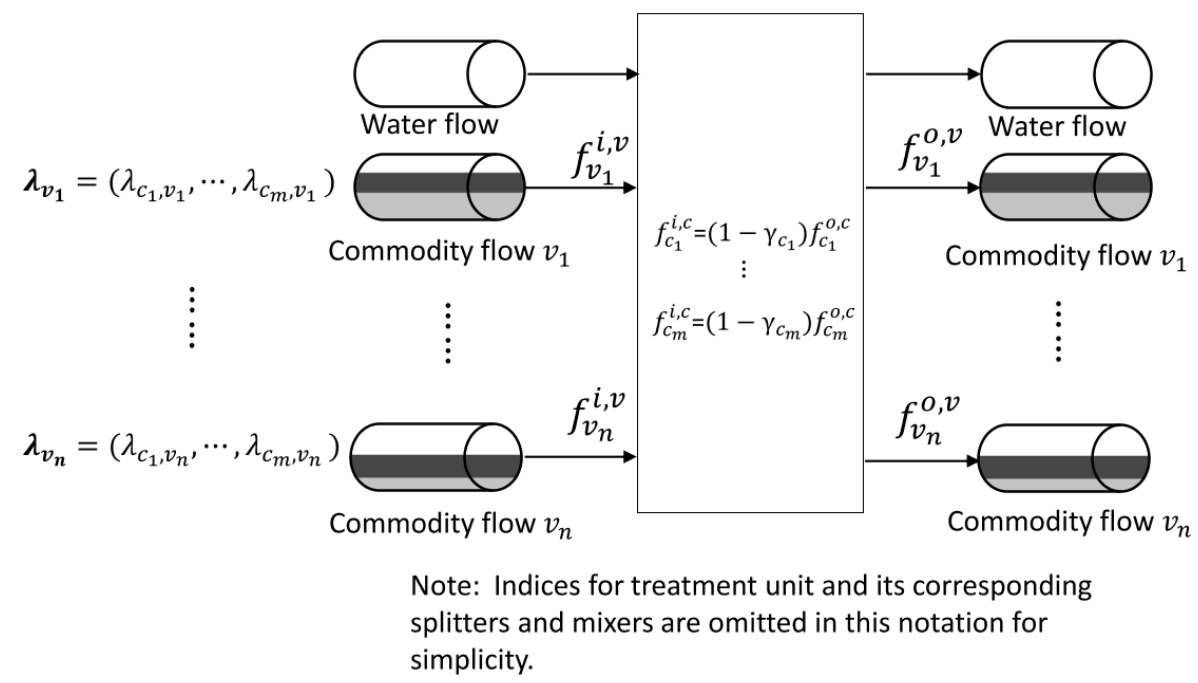

Figure 5: The commodity flows before and after the treatment unit $T_{1}$

Proposition 1. The incoming waste streams can be represented by commodities $v_{1}, v_{2}, \cdots, v_{n}$ if and only if $\exists \boldsymbol{F}^{v}=\left[\begin{array}{ccc}f_{v_{1}, s i_{1}} & \cdots & f_{v_{1}, s i_{w}} \\ \vdots & \ddots & \vdots \\ f_{v_{n}, s i_{1}} & \cdots & f_{v_{n}, s i_{w}}\end{array}\right]$ such that:

(a) $\boldsymbol{\Lambda} \boldsymbol{F}^{v}=\boldsymbol{\alpha}^{\boldsymbol{I N}}$

(b) $\left(\boldsymbol{F}^{v}\right)^{T} \mathbf{1} \leq \boldsymbol{\beta}^{\boldsymbol{I N}}$ (where $\mathbf{1}=(1,1, \cdots, 1) \in \mathbb{R}^{n}$ )

Proof. Condition (a) is to ensure that for each waste stream, the commodity flows that are used to represent the waste stream include the same total amount of each contaminant. Condition (b) implies that, there exist water flows of the waste streams $f_{v_{0}, s_{1}}, f_{v_{0}, s_{2}}, \cdots, f_{v_{0}, s_{w}} \geq$ 0 such that the following total mass balance holds for each waste stream:

$$
\sum_{v \in\left\{v_{0}, v_{1}, \cdots, v_{n}\right\}} f_{v, s}=\beta_{s}^{I N}, \quad \forall s \in\left\{s i_{1}, s i_{2}, \cdots, s i_{w}\right\} .
$$

Second, the selected group of commodities should be able to represent both the inlet flow and the outlet flow of a treatment unit. Figure 5 illustrates the flow rates of the same group of commodities for a treatment unit. For convenience of discussion, we define contaminant 
conversion matrix for the treatment unit:

$$
\boldsymbol{R}=\left[\begin{array}{lll}
1-\gamma_{c_{1}} & & \\
& \ddots & \\
& & 1-\gamma_{c_{m}}
\end{array}\right]
$$

The following proposition states the condition under which the outlet flow of a treatment unit can be represented by the same group of commodities that are used for the inlet flow.

Proposition 2. Consider a treatment unit whose inlet flow is represented by commodities $v_{1}, v_{2}, \cdots, v_{n}$ with flow rates $\boldsymbol{f}^{i, v}=\left(f_{v_{1}}^{i, v}, f_{v_{2}}^{i, v}, \cdots, f_{v_{n}}^{i, v}\right)$. The outlet flow of the treatment unit can be represented by the same commodities if and only if $\exists \boldsymbol{f}^{o, v}=\left(f_{v_{1}}^{o, v}, f_{v_{2}}^{o, v}, \cdots, f_{v_{n}}^{o, v}\right)$ such that $\boldsymbol{\Lambda} \boldsymbol{f}^{o, v}=\boldsymbol{R} \boldsymbol{\Lambda} \boldsymbol{f}^{i, v}$.

Proof. Let $\boldsymbol{f}^{i, c}=\left(f_{c_{1}}^{i, c}, f_{c_{2}}^{i, c}, \cdots, f_{c_{m}}^{i, c}\right)$ be the contaminant flows entering the treatment unit, then $\boldsymbol{f}^{i, c}=\boldsymbol{\Lambda} \boldsymbol{f}^{i, v}$. Let $\boldsymbol{f}^{o, c}=\left(f_{c_{1}}^{o, c}, f_{c_{2}}^{o, c}, \cdots, f_{c_{m}}^{o, c}\right)$ be contaminant flows leaving the treatment unit, then according to the definition of contaminant conversion matrix, $f^{o, c}=$ $\boldsymbol{R} \boldsymbol{f}^{i, c}=\boldsymbol{R} \boldsymbol{\Lambda} \boldsymbol{f}^{i, v}$.

On the other hand, let $\boldsymbol{f}^{o, v}=\left(f_{v_{1}}^{o, v}, f_{v_{2}}^{o, v}, \cdots, f_{v_{n}}^{o, v}\right)$ be the flow rates of the commodities for the outlet flow, then $\boldsymbol{f}^{o, c}=\boldsymbol{\Lambda} \boldsymbol{f}^{o, v}$. Therefore, $\boldsymbol{\Lambda} \boldsymbol{f}^{o, v}=\boldsymbol{R} \boldsymbol{\Lambda} \boldsymbol{f}^{i, v}\left(=\boldsymbol{f}^{o, c}\right)$ has to hold.

If for any treatment unit, all contaminants have the same removal ratio, i.e., $\gamma_{c_{1}}=\gamma_{c_{2}}=$ $\cdots=\gamma_{c_{m}}=\gamma$, then the condition in Proposition 2 becomes

$$
\boldsymbol{\Lambda} \boldsymbol{f}^{o, v}=\boldsymbol{\Lambda} \boldsymbol{R} \boldsymbol{f}^{i, v}=\boldsymbol{\Lambda} \boldsymbol{I}(1-\gamma) \boldsymbol{f}^{i, v}=\boldsymbol{\Lambda}\left((1-\gamma) \boldsymbol{f}^{i, v}\right)
$$

Therefore, the condition in Proposition 2 always holds no matter what selected commodities are. A generalized pooling network can be viewed as a special treatment network where all treatment units have zero removal ratios for all components. So we do not need to check the condition in Proposition 2 for GPP problems.

If at least one treatment unit has two different removal ratios, then whether the condition in Proposition 2 holds depends on $\Lambda$ (and therefore the selected commodities). In order 
for linear equation system $\boldsymbol{\Lambda} \boldsymbol{f}^{o, v}=\boldsymbol{R} \boldsymbol{\Lambda} \boldsymbol{f}^{i, v}$ to have a solution for any right-hand-side value, $m \leq \operatorname{dim}\left(\boldsymbol{f}^{o, v}\right)=n$. On the other hand, the number of selected commodities $n$ is proportional to how many bilinear terms are involved in the optimization (which can be seen in the next section), so we hope $n$ to be as small as possible in order for efficient global optimization. Therefore, we should select the commodities such that $\operatorname{rank}(\Lambda)=n$. Since $\operatorname{rank}(\Lambda) \leq m, n=m=\operatorname{rank}(\Lambda)$. In this case, $\boldsymbol{f}^{o, v}$ can be determined uniquely from $\boldsymbol{f}^{i, v}$ by

$$
\boldsymbol{f}^{o, v}=\boldsymbol{\Lambda}^{-1} \boldsymbol{R} \boldsymbol{\Lambda} \boldsymbol{f}^{i, v}=\boldsymbol{R}^{\prime} \boldsymbol{f}^{i, v}
$$

where

$$
\boldsymbol{R}^{\prime}=\left[\begin{array}{ccc}
r_{v_{1}, v_{1}}^{\prime} & \cdots & r_{v_{1}, v_{n}}^{\prime} \\
\vdots & \ddots & \vdots \\
r_{v_{n}, v_{1}}^{\prime} & \cdots & r_{v_{n}, v_{n}}^{\prime}
\end{array}\right]
$$

is called commodity conversion matrix. Note that in [28], the commodity flows are the total flows entering the sources, for which $\boldsymbol{\Lambda}$ may not be square and invertible. When using the demand commodity flows as in [29], $\boldsymbol{\Lambda}$ is not constant, so even if $\boldsymbol{\Lambda}$ is invertible, the strong nonlinear relationship between $\boldsymbol{f}^{o, v}$ and $\boldsymbol{f}^{i, v}$ will overly complicate the optimization problem.

Obviously, if the inlet flow of a splitter or a mixer can be represented by a set of commodities, then the outlet flow can also be represented by the same set of commodities. Therefore, the selected commodities can be used to represent all streams in a wastewater treatment network if and only if they satisfy the conditions in Propositions 1 and 2.

\subsection{Single Contaminant Commodity Flows}

A straightforward way to select commodities such that $\boldsymbol{\Lambda}$ is invertible is to use single contaminant commodities. A single contaminant commodity is a commodity that contains mixture of one contaminant and water. For contaminants $c_{1}, c_{2}, \cdots, c_{m}$, define single con- 
taminant commodities $v_{1}, v_{2}, \cdots, v_{m}$ such that

$$
\boldsymbol{\Lambda}=\left[\begin{array}{ccc}
\lambda_{c_{1}, v_{1}} & \cdots & 0 \\
\vdots & \ddots & \vdots \\
0 & \cdots & \lambda_{c_{m}, v_{m}}
\end{array}\right]
$$

The conditions in Proposition 1 can be simplified in the following condition:

$$
\left(\alpha^{I N}\right)^{T} \Lambda^{-1} 1 \leq \beta^{I N}
$$

This condition can be satisfied if the diagonal entries of $\boldsymbol{\Lambda}$ are sufficiently large. However, the diagonal entries of $\boldsymbol{\Lambda}$ cannot be too large because otherwise the commodity flow rates $f_{v, s i}$ would be very small and cause an ill-conditioned optimization problem. We also define null commodity $v_{0}$, which is pure water or a mixture of water and components that are not of interest.

In order to demonstrate the idea of single contaminant commodities, we show the optimal solutions of the problem instance Ex7 in the case study for the contaminant flow formulation and the commodity flow formulation, in Fig. 6 and 7 respectively. In Fig. 6, values along each pipeline are total flow rates $(\mathrm{t} / \mathrm{h})$ and contaminant flow rates $(\mathrm{kg} / \mathrm{h})$ in the pipeline, while in Fig 7 , the values are commodity flow rates $(\mathrm{t} / \mathrm{h})$ in the pipeline. The commodity composition matrix for the commodity flow formulation is

$$
\boldsymbol{\Lambda}=\left[\begin{array}{lll}
3 & 0 & 0 \\
0 & 5 & 0 \\
0 & 0 & 1
\end{array}\right] \quad\left(10^{3} p p m\right)
$$

Consider the waste stream that enters splitter $s i_{1}$. In the commodity flow formulation, it is represented as a mixture of:

$4 \mathrm{t} / \mathrm{h}$ of $v_{1}, 2 \mathrm{t} / \mathrm{h}$ of $v_{2}, 10 \mathrm{t} / \mathrm{h}$ of $v_{3}, 4 \mathrm{t} / \mathrm{h}$ of null commodity $v_{0}$. 


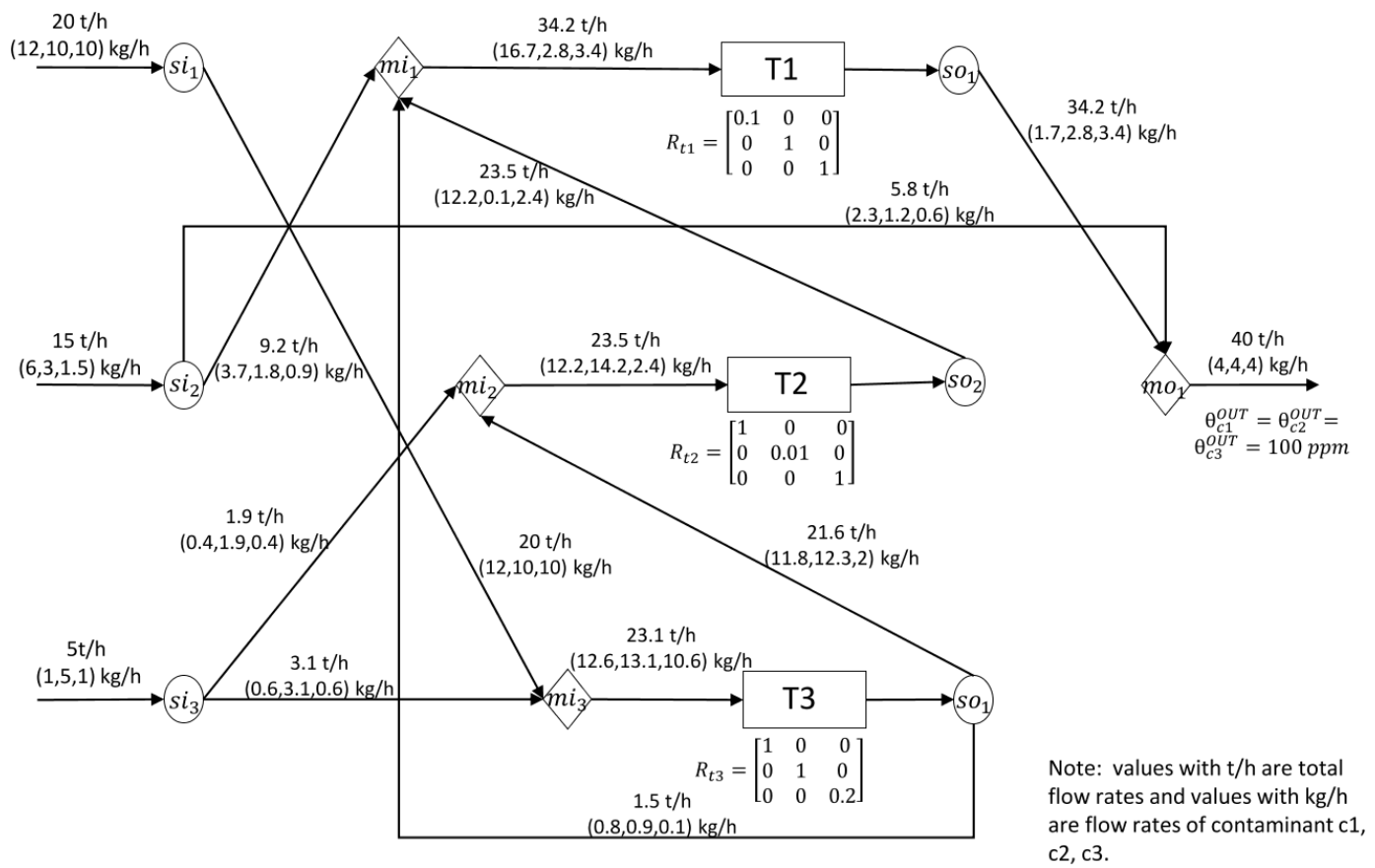

Figure 6: Optimal solution of the contaminant flow formulation of Ex7

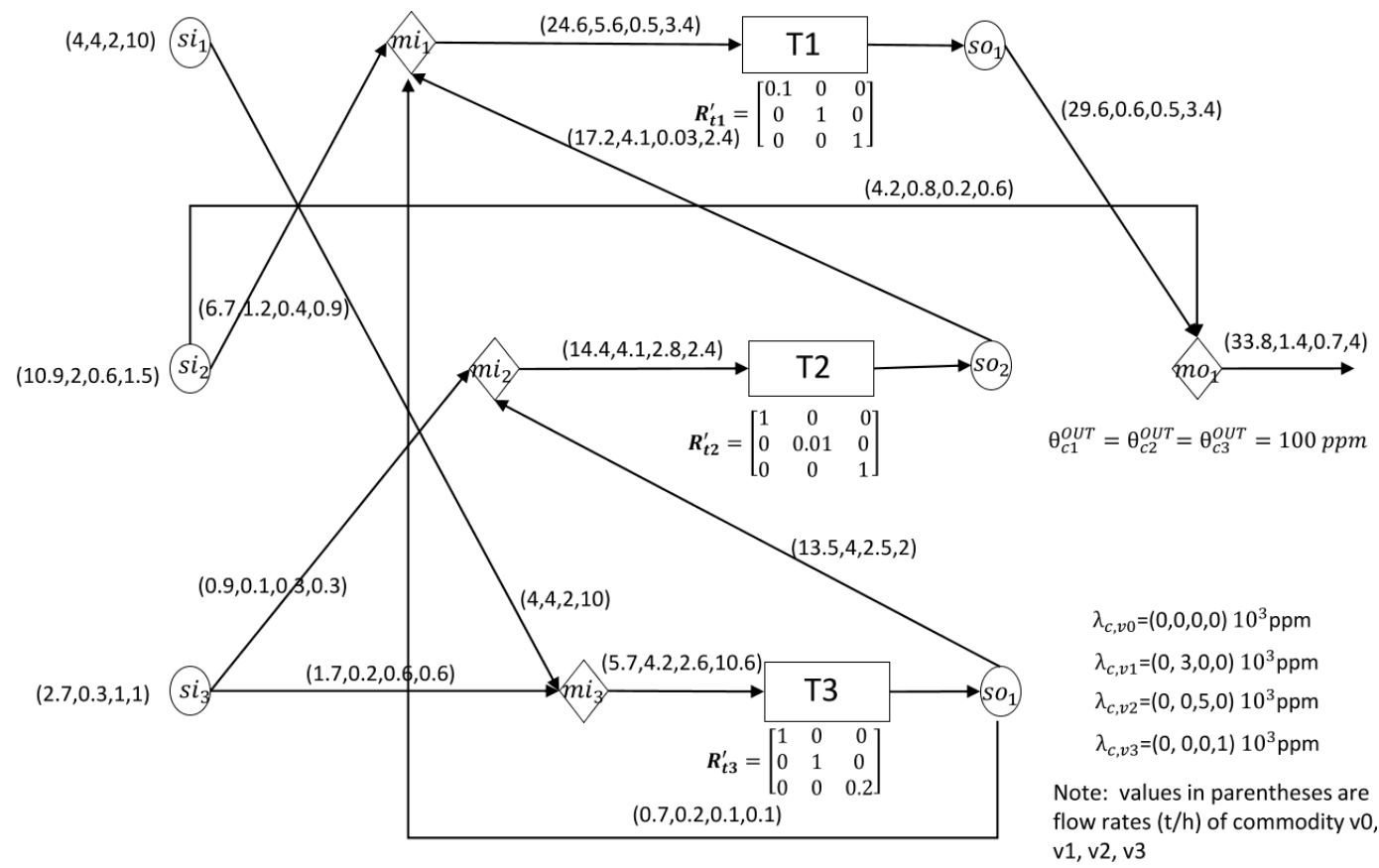

Figure 7: Optimal solution of the commodity flow formulation of Ex7 
According to the commodity compositions in $\boldsymbol{\Lambda}$, this mixture amounts to:

total waste stream flow rate: $4 \mathrm{t} / \mathrm{h}+2 \mathrm{t} / \mathrm{h}+10 \mathrm{t} / \mathrm{h}+4 \mathrm{t} / \mathrm{h}=20 \mathrm{t} / \mathrm{h}$,

flow rate of contaminant $c_{1}: 4 \mathrm{t} / \mathrm{h} \times 3 \times 10^{3} \mathrm{ppm}=12 \times 10^{-3} \mathrm{t} / \mathrm{h}=12 \mathrm{~kg} / \mathrm{h}$,

flow rate of contaminant $c_{2}: 2 \mathrm{t} / \mathrm{h} \times 5 \times 10^{3} \mathrm{ppm}=10 \times 10^{-3} \mathrm{t} / \mathrm{h}=10 \mathrm{~kg} / \mathrm{h}$,

flow rate of contaminant $c_{3}: 10 \mathrm{t} / \mathrm{h} \times 10^{3} \mathrm{ppm}=10 \times 10^{-3} \mathrm{t} / \mathrm{h}=10 \mathrm{~kg} / \mathrm{h}$,

which are same to the values in the contaminant flow formulation for the same waste stream. Also consider the inlet and outlet flows of treatment unit T1. In this treatment unit, $90 \%$ of contaminant $c_{1}$ is removed and other contaminants are unchanged. In Fig. 6 for the contaminant flow formulation, contaminant $c_{1}$ decreases from $16.7 \mathrm{~kg} / \mathrm{h}$ to $1.7 \mathrm{~kg} / \mathrm{h}$ through the treatment and the other two contaminant flows are unchanged. The total flow is also unchanged because the amount of removed mass is very small compared to the total mass flow. For the commodity flow formulation, the commodity conversion matrix that associate the inlet and outlet commodity flows is

$$
\boldsymbol{R}^{\prime}=\boldsymbol{\Lambda}^{-1} \boldsymbol{R} \Lambda=\left[\begin{array}{ccc}
0.1 & 0 & 0 \\
0 & 0 & 0 \\
0 & 0 & 0
\end{array}\right]
$$

so commodity $v_{1}$ decreases from $5.6 \mathrm{t} / \mathrm{h}$ to $0.6 \mathrm{t} / \mathrm{h}$. The $5 \mathrm{t} / \mathrm{h}$ of commodity $v_{1}$ is not removed from the network, but converted into the same amount of null commodity (because the removed mass is negligible), so $v_{0}$ increases from $24.6 \mathrm{t} / \mathrm{h}$ to $29.6 \mathrm{t} / \mathrm{h}$. Commodities $v_{1}$ and $v_{2}$ are unchanged through the treatment.

\section{A Multi-Commodity Flow Formulation for WWTN}

In this section, we present a multi-commodity flow formulation based on the commodity flow representation of streams discussed before. Here we only consider the situation in which at least one treatment unit has multiple different removal ratios. This is because most 
practical problems are in this situation, and for those not in this situation, the outlet flows of a treatment unit are simply constant fractions of the inlet flows and therefore the formulation is much easier. In this situation, we will select commodities such that $\boldsymbol{\Lambda}$ is constant, square and invertible, in order for efficient global optimization. Let $V=\left\{v_{1}, \cdots, v_{n}\right\}$ be the set of selected commodities that satisfy the conditions in Propositions 1 and 2, and let $v_{0}$ be the null commodity. In order to represent the waste streams with the commodities, the commodity flow rates $f_{v_{0}, s i}, f_{v_{1}, s i}, \cdots, f_{v_{n}, s i}$ must satisfy:

$$
\begin{gathered}
\sum_{v \in V \cup\left\{v_{0}\right\}} f_{v, s i}=\beta_{s i}^{I N}, \quad \forall s i \in S_{I} \\
\sum_{v \in V} f_{v, s i} \lambda_{c, v}=\alpha_{c, s i}^{I N}, \quad \forall s i \in S_{I}, c \in C
\end{gathered}
$$

The conditions in Proposition 1 ensure that such commodity flows exist, and $\boldsymbol{\Lambda}$ being invertible implies that such commodity flows can be uniquely determined from (PCC-2) with the given $\boldsymbol{\Lambda}$.

The proposed multi-commodity flow formulation is given as the following MCF formu- 
lation:

\section{The MCF Formulation}

$$
\begin{aligned}
& \min \sum_{t \in T} F_{t}^{T} \\
& \text { s.t. } f_{v, s i} x_{s i, m}=f_{s i, m, v} \quad \forall s i \in S_{I}, m \in M, v \in V \cup\left\{v_{0}\right\} \\
& f_{t, s o, v}^{o} x_{s o, m}=f_{s o, m, v} \quad \forall(t, s o) \in A_{O}, m \in M, v \in V \cup\left\{v_{0}\right\}, \\
& \sum_{m \in M} x_{s, m}=1 \quad \forall s \in S \\
& \sum_{s \in S} f_{s, m i, v}=f_{m i, t, v}^{i} \quad \forall v \in V \cup\left\{v_{0}\right\},(m i, t) \in A_{I} \\
& F_{t}^{T}=\sum_{v \in V \cup\left\{v_{0}\right\}} f_{m i, t, v}^{i} \quad \forall(m i, t) \in A_{I} \\
& f_{t, s o, v}^{o}=\sum_{v^{\prime} \in V} r_{t, v, v^{\prime}}^{\prime} f_{m i, t, v^{\prime}}^{i} \quad \forall(m i, t) \in A_{I},(t, s o) \in A_{O}, v \in V \\
& f_{t, s o, v_{0}}^{o}=F_{t}^{T}-\sum_{v \in V} f_{t, s o, v}^{o} \quad \forall(m i, t) \in A_{I},(t, s o) \in A_{O} \\
& \sum_{s \in S} \sum_{v \in V} f_{s, m i, v} \lambda_{c, v} \leq F_{t}^{T} \theta_{t, c}^{I N} \quad \forall(m i, t) \in A_{I}, c \in C \\
& \sum_{s \in S} \sum_{v \in V} f_{s, m o, v} \lambda_{c, v} \leq \sum_{s i \in S_{I}} \beta_{s i}^{I N} \theta_{c}^{O U T} \quad \forall c \in C, m o \in M_{O} \\
& f_{t, s o, v}^{o}=\sum_{m \in M} f_{s o, m, v} \quad \forall(t, s o) \in A_{O}, v \in V \cup\left\{v_{0}\right\} \\
& 0 \leq f_{t, s o, v}^{o} \leq \sum_{v^{\prime} \in V} r_{t, v, v^{\prime}}^{\prime} \sum_{s i \in S_{I}} f_{s i, v^{\prime}} \quad \forall(t, s o) \in A_{O}, v \in V \\
& 0 \leq f_{t, s o, v_{0}}^{o} \leq \sum_{s i \in S_{I}} \beta_{s i}^{I N} \quad \forall(t, s o) \in A_{O} \\
& 0 \leq x_{s, m} \leq 1 \quad \forall s \in S, m \in M \\
& 0 \leq F_{t}^{T} \leq \eta_{t} \quad \forall t \in T
\end{aligned}
$$

The objective function of the formulation is same to that of the component flow based formulation presented in Section 2. Eq. (MCF-2)-(MCF-4) represent the commodity bal- 
ances at the splitters, where (MCF-2) represents linear equations because $f_{s i, v}$ is a constant that is calculated from Eq. (PCC-1) and (PCC-2). Eq. (MCF-5) and (MCF-6) represent commodity balances at the inlet mixers. Eq. (MCF-7) represents balance equations of $v_{1}, v_{2}, \cdots, v_{n}$ at each treatment unit, which is essentially the aforementioned conversion relationship $\boldsymbol{f}^{o, v}=\boldsymbol{R}^{\prime} \boldsymbol{f}^{i, v}$. Eq. (MCF-8) represents the balance equation of the null commodity at each treatment unit; with the assumption that the mass of the removed contaminants is negligible, the outlet null commodity flow equals to the total outlet flow minus the sum of outlet flows of other commodities. Eq. (MCF-9) and (MCF-10) represent the quality constraints at the inlet mixers and at the outlet mixer, respectively. Eq. (MCF11) represents strengthening constraints obtained via summing (MCF-3) over $m \in M$. Eq. (MCF-12)-(MCF-14) provide bounds on the variables that appear in the bilinear terms. Eq. (MCF-15) enforces the total flow thought each treatment unit to be limited by the treatment capacity. The nonlinearity of the formulation comes solely from the bilinear equations in Eq. (MCF-3).

Next, we discuss the strength of the MCF formulation. We will prove that this formulation is at least as strong as the $\mathrm{C}$ formulation presented in Section 2, in the sense that the optimal objective value of the convex relaxation of the MCF formulation is no lower than that of the $\mathrm{C}$ formulation. The convex relaxation problem of $(\mathrm{MCF})$ can be obtained by replacing Eq. (MCF-3) with the convex hull relaxations [33], and the resulting formulation 
is expressed as the the following R-MCF problem:

\section{The R-MCF Formulation}

$$
\begin{aligned}
& \min (M C F-1) \\
& \text { s.t. }(M C F-2),(M C F-4)-(M C F-11),(M C F-14) \text {, } \\
& f_{s o, m, v} \geq \bar{x}_{s o, m} f_{t, s o, v}^{o}+x_{s o, m} \bar{f}_{t, s o, v}^{o}-\bar{x}_{s o, m} \bar{f}_{t, s o, v}^{o}, \quad \forall s o \in S_{O}, m \in M, v \in V \\
& f_{s o, m, v} \geq \underline{x}_{s o, m} f_{t, s o, v}^{o}+x_{s o, m} \underline{f}_{t, s o, v}^{o}-\underline{x}_{s o, m} \underline{f}_{t, s o, v}^{o}, \quad \forall s o \in S_{O}, m \in M, v \in V \\
& f_{s o, m, v} \leq \underline{x}_{s o, m} f_{t, s o, v}^{o}+x_{s o, m} \bar{f}_{t, s o, v}^{o}-\underline{x}_{s o, m} \bar{f}_{t, s o, v}^{o}, \quad \forall s o \in S_{O}, m \in M, v \in V \\
& f_{s o, m, v} \leq \bar{x}_{s o, m} f_{t, s o, v}^{o}+x_{s o, m} \underline{f}_{t, s o, v}^{o}-\bar{x}_{s o, m} \underline{f}_{t, s o, v}^{o} \quad \forall s o \in S_{O}, m \in M, v \in V \\
& \underline{f}_{t, s o, v}^{o} \leq f_{t, s o, v}^{o} \leq \bar{f}_{t, s o, v}^{o}, \quad \forall v \in V \\
& \underline{f}_{t, s o, v_{0}}^{o} \leq f_{t, s o, v_{0}}^{o} \leq \bar{f}_{t, s o, v_{0}}^{o} \\
& \underline{F}_{t}^{T} \leq F_{t}^{T} \leq \bar{F}_{t}^{T}, \quad \forall t \in T
\end{aligned}
$$

where the bar over or below a symbol indicates that it represents the upper or lower bound of the variable. Similarly, the convex relaxation of the C formulation, called the R-C problem, 
can be expressed as:

\section{The R-C Formulation}

$$
\begin{aligned}
& \min (C-1) \\
& \text { s.t. }(C-2)-(C-3),(C-6)-(C-13),(C-16) \\
& f_{s o, m, c} \geq \bar{x}_{s o, m} f_{t, s o, c}^{o}+x_{s o, m} \bar{f}_{t, s o, c}^{o}-\bar{x}_{s o, m} \bar{f}_{t, s o, c}^{o}, \quad \forall s o \in S_{O}, m \in M, c \in C \\
& \text { (R-C4-1) } \\
& f_{s o, m, c} \geq \underline{x}_{s o, m} f_{t, s o, c}^{o}+x_{s o, m} \underline{f}_{t, s o, c}^{o}-\underline{x}_{s o, m} \underline{f}_{t, s o, c}^{o}, \quad \forall s o \in S_{O}, m \in M, c \in C \\
& f_{s o, m, c} \leq \underline{x}_{s o, m} f_{t, s o, c}^{o}+x_{s o, m} \bar{f}_{t, s o, c}^{o}-\underline{x}_{s o, m} \bar{f}_{t, s o, c}^{o}, \quad \forall s o \in S_{O}, m \in M, c \in C \\
& f_{s o, m, c} \leq \bar{x}_{s o, m} f_{t, s o, c}^{o}+x_{s o, m} \underline{f}_{t, s o, c}^{o}-\bar{x}_{s o, m} \underline{f}_{t, s o, c}^{o}, \quad \forall s o \in S_{O}, m \in M, c \in C \\
& F_{s o, m} \geq \bar{x}_{s o, m} F_{t}^{T}+x_{s o, m} \bar{F}_{t}^{T}-\bar{x}_{s o, m} \bar{F}_{t}^{T}, \quad \forall s o \in S_{O}, m \in M \\
& F_{s o, m} \geq \underline{x}_{s o, m} F_{t}^{T}+x_{s o, m} \underline{F}_{t}^{T}-\underline{x}_{s o, m} \underline{F}_{t}^{T}, \quad \forall s o \in S_{O}, m \in M \\
& F_{s o, m} \leq \underline{x}_{s o, m} F_{t}^{T}+x_{s o, m} \bar{F}_{t}^{T}-\underline{x}_{s o, m} \bar{F}_{t}^{T}, \quad \forall s o \in S_{O}, m \in M \\
& F_{s o, m} \leq \bar{x}_{s o, m} F_{t}^{T}+x_{s o, m} \underline{F}_{t}^{T}-\bar{x}_{s o, m} \underline{F}_{t}^{T}, \quad \forall s o \in S_{O}, m \in M \\
& \underline{f}_{t, s o, c}^{o} \leq f_{t, s o, c}^{o} \leq \bar{f}_{t, s o, c}^{o}, \quad \forall(t, s o) \in A_{O}, c \in C \\
& \underline{F}_{t}^{T} \leq F_{t}^{T} \leq \bar{F}_{t}^{T}, \quad \forall t \in T
\end{aligned}
$$

In (R-MCF), the variables to be branched on in the solution procedure are $x_{s o, m}, f_{t, s o, v}^{o}$, and in (R-C) these variables are $x_{s o, m}, f_{t, s o, c}^{o}$ and $F_{t}^{T}$. We make the following bound 
consistency assumptions for a fair comparison of the two relaxations:

$$
\begin{array}{r}
\overline{\boldsymbol{f}}_{t, s o}^{o, c}=\boldsymbol{\Lambda} \overline{\boldsymbol{f}}_{t, s o}^{o, v}, \quad \forall(t, s o) \in A_{O} \\
\underline{\boldsymbol{f}}_{t, s o}^{o, c}=\boldsymbol{\Lambda} \underline{\boldsymbol{f}}_{t, s o}^{o, v}, \quad \forall(t, s o) \in A_{O} \\
\bar{F}_{t}^{T}=\sum_{v \in V \cup\left\{v_{o}\right\}} \bar{f}_{t, s o, v}^{o}, \quad \forall(t, s o) \in A_{O} \\
\underline{F}_{t}^{T}=\sum_{v \in V \cup\left\{v_{o}\right\}} \underline{f}_{t, s o, v}^{o}, \quad \forall(t, s o) \in A_{O}
\end{array}
$$

where vector $\boldsymbol{f}_{t, \boldsymbol{s} o}^{o, \boldsymbol{c}}$ includes all $f_{t, s o, c}^{o}$ in (R-C), and $\boldsymbol{f}_{\boldsymbol{t}, \boldsymbol{s o}}^{\boldsymbol{o} \boldsymbol{v}}$ includes all $f_{t, s o, v}^{o}$ in (R-MCF) except for the null commodity.

Proposition 3. Let $O b j_{R-M C F}$ and $O b j_{R-C}$ be the optimal objective values of problem $(R-M C F)$ and problem $(R-C)$, respectively, then

$$
O b j_{R-M C F} \geq O b j_{R-C} .
$$

Proof. Since the two relaxation problems have the same objective function, it suffices to prove that any $F_{t}^{T}(\forall t \in T)$ that is feasible for problem (R-MCF) is also feasible for problem (R-C). For $F_{t}^{T}(\forall t \in T)$ that is feasible for problem (R-MCF), there exist $f_{s, m, v}, x_{s, m}$, $f_{t, s o, v}^{o}, f_{m i, t, v}^{i}\left(\forall s \in S, m \in M, v \in V \cup\left\{v_{0}\right\},(t, s o) \in A_{O},(m i, t) \in A_{I}, t \in T\right)$ such that all constraints in (R-MCF) are satisfied. Note that $f_{v, s i}\left(\forall s i \in S_{I}, v \in V \cup\left\{v_{0}\right\}\right)$ in (R-MCF) 
are parameters satisfying Eq. (PCC-1) and (PCC-2). Now we define

$$
\begin{aligned}
f_{s, m, c} & =\sum_{v \in V} f_{s, m, v} \lambda_{c, v}, \quad \forall s \in S, m \in M, c \in C \\
f_{t, s o, c}^{o} & =\sum_{v \in V} f_{t, s o, v}^{o} \lambda_{c, v}, \quad \forall(t, s o) \in A_{O}, c \in C \\
f_{m i, t, c}^{i} & =\sum_{v \in V} f_{m i, t, v}^{i} \lambda_{c, v}, \quad \forall(m i, t) \in A_{I}, c \in C \\
F_{s, m} & =\sum_{v \in V \cup\left\{v_{0}\right\}} f_{s, m, v}, \quad \forall s \in S, m \in M
\end{aligned}
$$

and show that these new quantities together with the $x_{s, m}, F_{t}^{T}(\forall s \in S, m \in M, t \in T)$ satisfy all constraints in (R-C), and therefore the $F_{t}^{T}(\forall t \in T)$ is also feasible for problem (R-C).

Since we are considering the same $x_{s, m}, F_{t}^{T}$ for both relaxation problems, (C-6), (C-15), and (C-16) are implied by (MCF-4), (MCF-14), and (MCF-15).

(LT-4), (MCF-2), and (PCC-1) imply (C-2):

$$
F_{s i, m}=\sum_{v \in V \cup\left\{v_{0}\right\}} f_{s i, m, v}=\sum_{v \in V \cup\left\{v_{0}\right\}} f_{v, s i} x_{s i, m}=\beta_{s i}^{I N} x_{s i, m}
$$

(LT-1), (MCF-2), and (PCC-2) imply (C-3):

$$
f_{s i, m, c}=\sum_{v \in V} f_{s i, m, v} \lambda_{c, v}=\sum_{v \in V} f_{v, s i} x_{s i, m} \lambda_{c, v}=\alpha_{c, s i}^{I N} x_{s i, m} .
$$

(LT-3), (MCF-5), and (LT-1) imply (C-7):

$$
f_{m i, t, c}^{i}=\sum_{v \in V} f_{m i, t, v}^{i} \lambda_{c, v}=\sum_{v \in V} \sum_{s \in S} f_{s, m i, v} \lambda_{c, v}=\sum_{s \in S} \sum_{v \in V} f_{s, m i, v} \lambda_{c, v}=\sum_{s \in S} f_{s, m i, c} .
$$

(MCF-6), (MCF-5), and (LT-4) imply (C-8):

$$
F_{t}^{T}=\sum_{v \in V \cup\left\{v_{0}\right\}} f_{m i, t, v}^{i}=\sum_{v \in V \cup\left\{v_{0}\right\}} \sum_{s \in S} f_{s, m i, v}=\sum_{s \in S} \sum_{v \in V \cup\left\{v_{0}\right\}} f_{s, m i, v}=\sum_{s \in S} F_{s, m i} .
$$


(MCF-7), (LT-2), (LT-3) imply (C-9). In order to show this, define vectors $\boldsymbol{f}_{\boldsymbol{m} \boldsymbol{i , t}, \boldsymbol{c}}^{\boldsymbol{i} \boldsymbol{c}}=$ $\left(f_{m i, t, c_{1}}^{i}, \cdots, f_{m i, t, c_{m}}^{i}\right), \boldsymbol{f}_{\boldsymbol{m i , t}}^{i, \boldsymbol{v}}=\left(f_{m i, t, v_{1}}^{i}, \cdots, f_{m i, t, v_{n}}^{i}\right), \boldsymbol{f}_{\boldsymbol{t}, \boldsymbol{s o}}^{\boldsymbol{o}, \boldsymbol{c}}=\left(f_{t, s o, c_{1}}^{o}, \cdots, f_{t, s o, c_{m}}^{o}\right), \boldsymbol{f}_{\boldsymbol{t}, \boldsymbol{s o}}^{\boldsymbol{o}, \boldsymbol{v}}=$ $\left(f_{t, s o, v_{1}}^{o}, \cdots, f_{t, s o, v_{n}}^{o}\right)$, then (MCF-7), (LT-2) and (LT-3) can be rewritten as:

$$
\begin{gathered}
f_{t, s o}^{o, v}=R^{\prime} f_{m i, t}^{i, v} \\
f_{t, s o}^{o, c}=\Lambda f_{t, s o}^{o, v} \\
f_{m i, t}^{i, c}=\Lambda f_{m i, t}^{i, v} .
\end{gathered}
$$

Therefore,

$$
f_{t, s o}^{o, c}=\Lambda f_{t, s o}^{o, v}=\Lambda R^{\prime} f_{m i, t}^{i, v}=\Lambda R^{\prime} \Lambda^{-1} f_{m i, t}^{i, c}=\Lambda\left(\Lambda^{-1} R \Lambda\right) \Lambda^{-1} f_{m i, t}^{i, c}=R f_{m i, t}^{i, c}
$$

According to the definition of contaminant conversion matrix $\boldsymbol{R}, \boldsymbol{f}_{\boldsymbol{t}, \boldsymbol{s o}}^{\boldsymbol{o}, \boldsymbol{c}}=\boldsymbol{R} \boldsymbol{f}_{\boldsymbol{m} \boldsymbol{i , t}}^{i, \boldsymbol{c}}$ is (C-9).

(C-10) and (C-11) are strengthening constraints obtained from (C-5) and (C-4), so they are also satisfied.

(LT-1) and (MCF-9) imply (C-12):

$$
\sum_{s \in S} f_{s, m i, c}=\sum_{s \in S} \sum_{v \in V} f_{s, m i, v} \lambda_{c, v} \leq F_{t}^{T} \theta_{t, c}^{I N}
$$

(LT-1) and (MCF-10) imply (C-13):

$$
\sum_{s \in S} f_{s, m o, c}=\sum_{s \in S} \sum_{v \in V} f_{s, m o, v} \lambda_{c, v} \leq F^{O U T} \theta_{c}^{O U T}
$$

According to (LT-1), (R-MCF3-1), (LT-2), and (BC-1), $\forall s o \in S_{O}, m \in M, c \in C$,

$$
\begin{aligned}
f_{s o, m, c} & =\sum_{v \in V} f_{s o, m, v} \lambda_{c, v} \\
& \geq \bar{x}_{s o, m} \sum_{v \in V} \lambda_{c, v} f_{t, s o, v}^{o}+x_{s o, m} \sum_{v \in V} \lambda_{c, v} \bar{f}_{t, s o, v}^{o}-\bar{x}_{s o, m} \sum_{v \in V} \lambda_{v, c} \bar{f}_{t, s o, v}^{o} \\
& =\bar{x}_{s o, m} f_{t, s o, c}^{o}+x_{s o, m} \bar{f}_{t, s o, c}^{o}-\bar{x}_{s o, m} \bar{f}_{t, s o, c}^{o},
\end{aligned}
$$


which is the relaxation constraint (R-C4-1). Similarly, we can prove that (R-C4-2)-(R-C4-4) are also satisfied.

(LT-4), (R-MCF3-1) imply:

$F_{s o, m}=\sum_{v \in V \cup\left\{v_{0}\right\}} f_{s o, m, v} \geq \bar{x}_{s o, m} \sum_{v \in V \cup\left\{v_{0}\right\}} f_{t, s o, v}^{o}+x_{s o, m} \sum_{v \in V \cup\left\{v_{0}\right\}} \bar{f}_{t, s o, v}^{o}-\bar{x}_{s o, m} \sum_{v \in V \cup\left\{v_{0}\right\}} \bar{f}_{t, s o, v}^{o}$.

From (MCF-7), and (MCF-8), $\sum_{v \in V \cup\left\{v_{0}\right\}} f_{t, s o, v}^{o}=F_{t}^{T}$, and together with (BC-3), we have

$$
F_{s o, m} \geq \bar{x}_{s o, m} F_{t}^{T}+x_{s o, m} \bar{F}_{t}^{T}-\bar{x}_{s o, m} \bar{F}_{t}^{T}
$$

so (R-C5-1) is satisfied. Similarly, we can prove (R-C5-2) -(R-C5-4).

Since $\boldsymbol{f}_{\boldsymbol{t}, \boldsymbol{s o}}^{\boldsymbol{o}, \boldsymbol{c}}=\boldsymbol{\Lambda} \boldsymbol{f}_{\boldsymbol{t}, \boldsymbol{s o}}^{\boldsymbol{o}, \boldsymbol{v}},(\mathrm{R}-\mathrm{MCF} 12),(\mathrm{BC}-1)$, and (BC-2) imply (R-C14). This completes the proof.

\section{Computational Study}

The goal of the computational study is to show the strength and the solution efficiency of the MCF formulation in comparison to the C formulation, via 16 WWTN instances from the literature. The simulation was performed on a virtual machine with 3-core $3.40 \mathrm{GHz}$ CPU, 4GB memory, and Ubuntu 16.02 operating system. All problems were formulated on GAMS 26.1.0 [34], and solved by SCIP 6.0 [35] that calls CPLEX 12.8.0 for solving linear programming (LP) subproblems. CPLEX 12.8.0 was also used for solving other LP problems needed in the study. The CPLEX solver was set to utilize the 6 threads provided by the 3 -core CPU allocated to the virtual machine. The run time limit was set to be 2 hours, and the relative and absolute termination tolerances were both $10^{-2}$.

The 16 WWTN instances are denoted as Ex1-Ex16. Ex1-Ex9 are from [31], and Ex10Ex16 are from [25]. Instances Ex9, Ex11-Ex13 and Ex15 can not be solved in the 2-hour time limit with either the $\mathrm{C}$ or the MCF formulation. Therefore, we modified these instances so that at least one of the formulations can solve each instance within the time limit and which formulation is better is more clear. This is how we modified these instances. For 
Ex9 only the first 2 contaminants were considered. For Ex11 and Ex12 the last treatment unit was removed. For Ex13 the last 7 treatment units were removed. For Ex15 $\gamma_{t 3, c 3}$ was changed from 0 to 0.9 .

In the computational study, we chose single contaminant commodities to represent streams for all problem instances. As mentioned in Section 3, single contaminant commodities always satisfy the condition in Proposition 2, and the conditions in Proposition 1 can be simplified as a simple inequality that is easy to check $\left(\left(\boldsymbol{\alpha}^{\boldsymbol{I N}}\right)^{\boldsymbol{T}} \boldsymbol{\Lambda}^{-\mathbf{1}} \mathbf{1} \leq \boldsymbol{\beta}^{\boldsymbol{I N}}\right)$. In this paper, we choose the following commodity compositions which satisfy the inequality for problem instances:

$$
\lambda_{c, v}=\max _{s i} 5 \alpha_{s i, c}^{I N} / \beta_{s i}^{I N}, \quad \forall v \neq v_{0}, c \in C
$$

\subsection{Computational Results}

We compare the computational performance of MCF formulation and $\mathrm{C}$ formulation by showing both the root gap and the solution time for the 16 problem instances. The root gap $\delta$ is defined as

$$
\delta=\frac{\left|\mathrm{Obj}_{\text {gop }}-\mathrm{Obj}_{\text {root }}\right|}{\left|\mathrm{Obj}_{\text {gop }}\right|} \times 100 \%
$$

where $\mathrm{Obj}_{g o p}$ is the optimal objective value of the optimization problem, and $\mathrm{Obj}_{\text {root }}$ is the optimal objective of the convex relaxation problem obtained at the root node of the branchand-bound search. We also investigate how optimality-based bound tightening (OBBT) may affect the performance of the two formulations. Specifically, we compare the two formulations for both the situation in which no OBBT is performed before sending the problem to SCIP and the situation in which no initial OBBT is performed.

Table 2 shows the details of the problem instances and the computational results. Columns 2-4 in the table provide features of the superstructures in the 16 instances, including the number of treatment units $(|T|)$, the number of inlet splitters $\left(\left|S_{I}\right|\right)$, and the number of contaminants $(|C|)$. Column 5 shows the number of bilinear terms for the instances. Note that the numbers of bilinear terms in the two formulations are same. For the $\mathrm{C}$ formulation, it is $n_{c}=\left|S_{O}\right| \times|M| \times(|C|+1)$. For the MCF formulation, it is $n_{M C F}=\left|S_{O}\right| \times|M| \times\left|V \cup\left\{v_{0}\right\}\right|$, and $|V|=|C|$ for single contaminant flows. Columns 6 
shows the optimal objective values. The subsequent columns compare the root gaps and the CPU seconds from the $\mathrm{C}$ and the MCF formulations. When there is no initial OBBT, the MCF formulation achieves a better root gap and better solution efficiency for all problem instances. When there is initial OBBT, both the $\mathrm{C}$ formulation and the MCF formulation achieve better root gap and CPU time, but the MCF formulation is still more efficient than the $\mathrm{C}$ formulation for most problem instances, especially for more difficult ones. For most problem instances, the root gap of the $\mathrm{C}$ formulation is same or very close to that of the MCF formulation, which may be because the $\mathrm{C}$ formulation benefits more from the initial OBBT. For easy problems, such as Ex1-Ex3, the two formulations render the same solution time. However, if the problem instance takes more time to solve (implying more branch-andbound nodes explored), such as Ex16, the MCF formulation is more advantageous because it is a stronger formulation not only at the root node, but also at all nodes. On average, the MCF formulation reduces the solution time by $42 \%$, and for Ex9, it reduces the solution time by nearly two orders of magnitudes.

\subsection{Optimal Solutions of Ex'}

For all problem instances, the $\mathrm{C}$ and the $\mathrm{MCF}$ formulations have the same optimal objective value that comes from the same optimal network structure. However, the two formulations use different stream representations, so they have different decision variables and optimal solution values. We demonstrate this through Ex7, whose solutions are already shown in Fig. 6 and Fig. 7 in Section 3.4. In both figures, waste stream 1 goes though all the three treatment units, only part of waste stream 3 goes through all the three treatment units, and waste stream 2 partly goes through treatment unit 1 and party goes to discharge without treatment. This is because waste stream 1 has the highest concentrations of contaminants.

In order to demonstrate the advantage of global optimization for the WWTN problem, we also solved the $\mathrm{C}$ and the MCF formulations of Ex7 using a local optimization solver IPOPT 3.12 [36]. Fig 8 and Fig 9 show the optimal network structures and objective values obtained by IPOPT with the two formulations. It can be seen that the two formulations lead to different local optimal network structures, and both structures are more complicated 


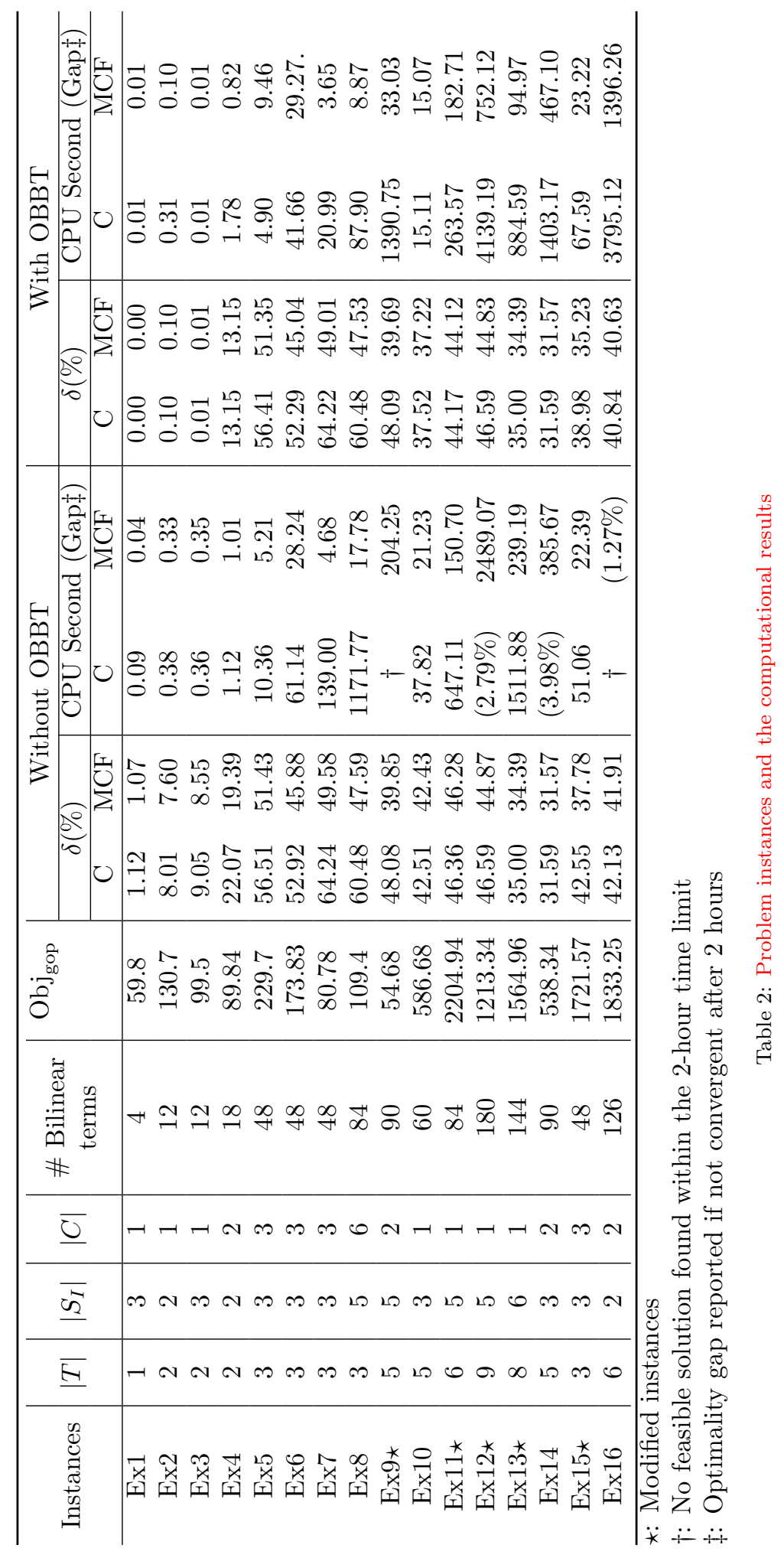




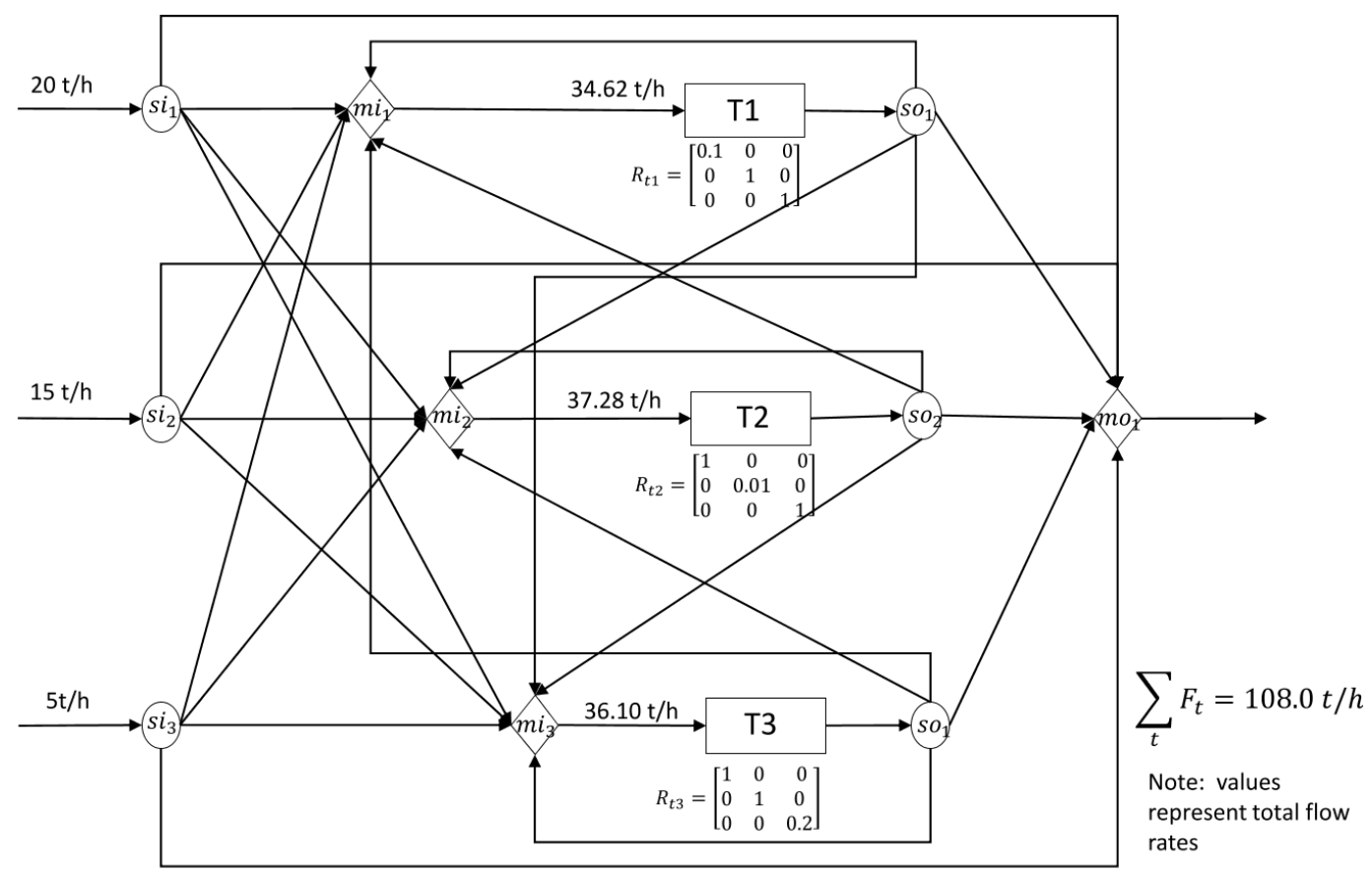

Figure 8: Local optimal solution of the $\mathrm{C}$ formulation of Ex7 (obtained by IPOPT)

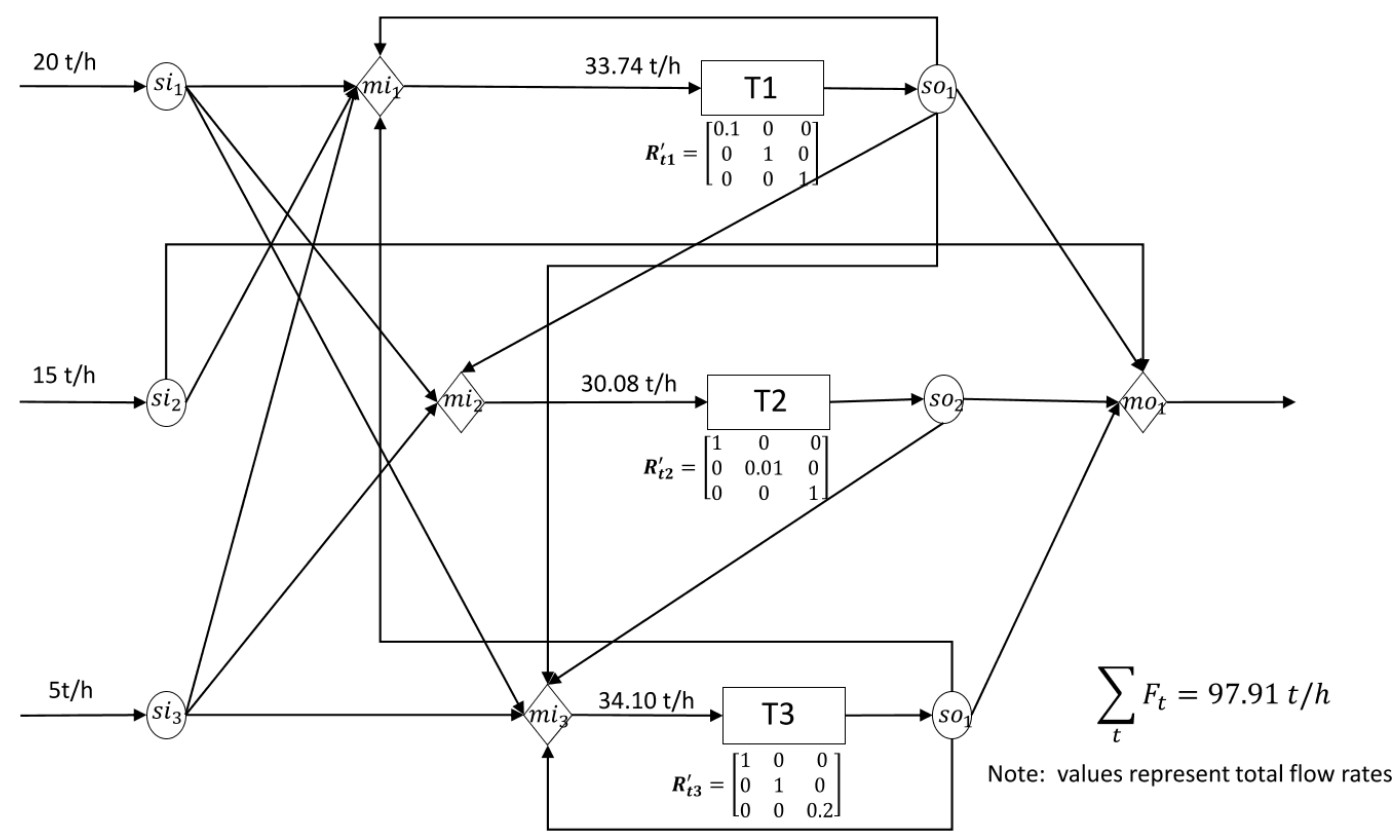

Figure 9: Local optimal solution of the MCF formulation of Ex7 (obtained by IPOPT) 
than the global optimal structure in Fig 6 and 7. The sum of the flows going through each of the treatment units is $108.0 \mathrm{t} / \mathrm{h}$ in the local solution of the $\mathrm{C}$ formulation, and $97.91 \mathrm{t} / \mathrm{h}$ in the local solution of the MCF formulation, and $80.78 \mathrm{t} / \mathrm{h}$ in the global solution. This indicates that, the local optimal solution renders $21.1 \%$ or $33.7 \%$ more operating cost than the global optimal solution.

\section{Concluding Remarks}

In this paper, we propose a multi-commodity flow formulation for the WWTN problem. We first generalize the concept of commodity in the classical multi-commodity flow formulations for the GPP, then discuss conditions under which a set of commodities are adequate for representing streams in a WWTN. We find that, in most cases, the commodity composition matrix $\Lambda$ needs to be invertible in order to represent the streams with the fewest commodities. A simple way to construct eligible commodities is to allow only one contaminant in one commodity, and this single-contaminant commodity strategy is adopted in the computational study. The computational results of 16 problem instances show the significant computational advantage of the proposed MCF formulation over the classical component based $\mathrm{C}$ formulation. For half of the problem instances, the solution procedure with the MCF formulation is 2-40 times faster than that with the $\mathrm{C}$ formulation. In addition, the global optimal solution can achieve significantly better economic performance than a local optimal solution. For problem instance Ex7, the local solution of the $\mathrm{C}$ or the MCF formulation renders $33.7 \%$ or $21.1 \%$ more operating cost than the global solution does.

Since there exist different types of multi-commodity flow formulations for the GPP, there may also exist other types of multi-commodity flow formulations for the WWTN problem. It will be interesting to develop these formulations and compare them with the MCF formulation proposed in this paper. We will also be interested in developing multicommodity flow formulations for the combined water use and water treatment network, and evaluating their computational advantages over the component based formulations. 


\section{Acknowledgement}

The authors are grateful to the financial support from Natural Sciences and Engineering Research Council of Canada through the Discovery Grant RGPIN 418411-13 and the Collaborative Research and Development Grant CRDPJ 485798-15.

\section{References}

[1] T. Sato, M. Qadir, S. Yamamoto, T. Endo, A. Zahoor, Global, regional, and country level need for data on wastewater generation, treatment, and use, Agricultural Water Management 130 (2013) 1-13.

[2] Z. D. Forshomi, A. Alva-Argaez, J. A. Bergerson, Optimal design of distributed effluent treatment systems in steam assisted gravity drainage oil sands operations, Journal of cleaner production 149 (2017) 1233-1248.

[3] S. Bandyopadhyay, M. D. Ghanekar, H. K. Pillai, Process water management, Industrial \& Engineering Chemistry Research 45 (15) (2006) 5287-5297.

[4] N. BENK, E. REV, Z. FONYO, The use of nonlinear programming to optimal water allocation, Chemical Engineering Communications 178 (1) (2000) 67-101.

[5] C.-T. Chang, B.-H. Li, Improved optimization strategies for generating practical waterusage and-treatment network structures, Industrial \& engineering chemistry research 44 (10) (2005) 3607-3618.

[6] R. Karuppiah, I. E. Grossmann, Global optimization for the synthesis of integrated water systems in chemical processes, Computers \& Chemical Engineering 30 (4) (2006) $650-673$.

[7] R. Karuppiah, I. E. Grossmann, Global optimization of multiscenario mixed integer nonlinear programming models arising in the synthesis of integrated water networks under uncertainty, Computers \& Chemical Engineering 32 (1-2) (2008) 145-160. 
[8] Y.-P. Wang, R. Smith, Design of distributed effluent treatment systems, Chemical Engineering Science 49 (18) (1994) 3127-3145.

[9] W.-C. J. Kuo, R. Smith, Effluent treatment system design, Chemical Engineering Science 52 (23) (1997) 4273-4290.

[10] D. C. Y. Foo, State-of-the-art review of pinch analysis techniques for water network synthesis, Industrial \& Engineering Chemistry Research 48 (11) (2009) 5125-5159.

[11] B. Galan, I. Grossmann, Optimal design of distributed wastewater treatment networks, Industrial \& engineering chemistry research 37 (10) (1998) 4036-4048.

[12] C.-H. Huang, C.-T. Chang, H.-C. Ling, C.-C. Chang, A mathematical programming model for water usage and treatment network design, Industrial \& Engineering Chemistry Research 38 (7) (1999) 2666-2679.

[13] L. Song, J. Du, S. Cai, P. Yao, A new method for designing water network based on variable removal ratio of treatment process, in: Computer Aided Chemical Engineering, Vol. 21, Elsevier, 2006, pp. 1783-1789.

[14] J. Jezowski, Review of water network design methods with literature annotations, Industrial \& Engineering Chemistry Research 49 (10) (2010) 4475-4516.

[15] P. M. Castro, J. P. Teles, A. Q. Novais, Linear program-based algorithm for the optimal design of wastewater treatment systems, Clean Technologies and Environmental Policy 11 (1) (2009) 83.

[16] J. Teles, P. M. Castro, A. Q. Novais, Lp-based solution strategies for the optimal design of industrial water networks with multiple contaminants, Chemical Engineering Science 63 (2) (2008) 376-394.

[17] R. Hernández-Suárez, J. Castellanos-Fernández, J. M. Zamora, Superstructure decomposition and parametric optimization approach for the synthesis of distributed wastewater treatment networks, Industrial \& Engineering Chemistry Research 43 (9) (2004) $2175-2191$. 
[18] G. Statyukha, O. Kvitka, I. Dzhygyrey, J. Jeżowski, A simple sequential approach for designing industrial wastewater treatment networks, Journal of Cleaner Production 16 (2) (2008) 215-224.

[19] M.-J. Tsai, C.-T. Chang, Water usage and treatment network design using genetic algorithms, Industrial \& engineering chemistry research 40 (22) (2001) 4874-4888.

[20] G. Poplewski, J. Jeżowski, A simultaneous approach for designing optimal wastewater treatment network, Chem. Eng. Trans 12 (2007) 321-326.

[21] J. M. Zamora, I. E. Grossmann, Continuous global optimization of structured process systems models, Computers \& chemical engineering 22 (12) (1998) 1749-1770.

[22] R. Karuppiah, K. C. Furman, I. E. Grossmann, Global optimization for scheduling refinery crude oil operations, Computers \& Chemical Engineering 32 (11) (2008) 27452766 .

[23] M. L. Bergamini, I. Grossmann, N. Scenna, P. Aguirre, An improved piecewise outerapproximation algorithm for the global optimization of minlp models involving concave and bilinear terms, Computers \& Chemical Engineering 32 (3) (2008) 477-493.

[24] J. P. Teles, P. M. Castro, H. A. Matos, Multi-parametric disaggregation technique for global optimization of polynomial programming problems, Journal of Global Optimization (2013) 1-25.

[25] J. P. Teles, P. M. Castro, H. A. Matos, Global optimization of water networks design using multiparametric disaggregation, Computers \& Chemical Engineering 40 (2012) $132-147$.

[26] L. Ting, P. M. Castro, L. Zhimin, Models and relaxations for the wastewater treatment design problem, Chemical Engineering Research and Design 106 (2016) 191-204.

[27] C. A. Haverly, Studies of the behavior of recursion for the pooling problem, ACM Sigmap Bulletin (25) (1978) 19-28. 
[28] M. Alfaki, D. Haugland, A multi-commodity flow formulation for the generalized pooling problem, Journal of Global Optimization 56 (3) (2013) 917.

[29] N. Boland, T. Kalinowski, F. Rigterink, New multi-commodity flow formulations for the pooling problem, Journal of Global Optimization 66 (4) (2016) 669-710.

[30] S. Doyle, R. Smith, Targeting water reuse with multiple contaminants, Process safety and environmental protection 75 (3) (1997) 181-189.

[31] P. M. Castro, H. A. Matos, A. Q. Novais, An efficient heuristic procedure for the optimal design of wastewater treatment systems, Resources, conservation and recycling 50 (2) (2007) 158-185.

[32] H. D. Sherali, A. Alameddine, A new reformulation-linearization technique for bilinear programming problems, Journal of Global optimization 2 (4) (1992) 379-410.

[33] G. P. McCormick, Computability of global solutions to factorable nonconvex programs: Part i-convex underestimating problems, Mathematical programming 10 (1) (1976) $147-175$.

[34] M. R. Bussieck, A. Meeraus, General algebraic modeling system (gams), in: Modeling languages in mathematical optimization, Springer, 2004, pp. 137-157.

[35] A. Gleixner, M. Bastubbe, L. Eifler, T. Gally, G. Gamrath, R. L. Gottwald, G. Hendel, C. Hojny, T. Koch, M. E. Lübbecke, S. J. Maher, M. Miltenberger, B. Müller, M. E. Pfetsch, C. Puchert, D. Rehfeldt, F. Schlösser, C. Schubert, F. Serrano, Y. Shinano, J. M. Viernickel, M. Walter, F. Wegscheider, J. T. Witt, J. Witzig, The SCIP Optimization Suite 6.0, ZIB-Report 18-26, Zuse Institute Berlin (July 2018).

URL http://nbn-resolving.de/urn:nbn:de:0297-zib-69361

[36] A. Wächter, L. T. Biegler, On the implementation of an interior-point filter line-search algorithm for large-scale nonlinear programming, Mathematical Programming 106 (1) (2006) 25-57. 


\section{Appendix: Equivalence of the Contaminant Flow Formulation in the Paper and}

the One in Castro et al. [31]

\begin{tabular}{|c|c|c|}
\hline & $\begin{array}{l}\text { The contaminant flow } \\
\text { formulation in this paper }\end{array}$ & Formulation M2 in [31] \\
\hline Objective & C-1 & M2-1 \\
\hline \multirow{9}{*}{$\begin{array}{l}\text { Material } \\
\text { Balance } \\
\text { Constraints }\end{array}$} & $\mathrm{C}-4$ & M2-19,M2-20 \\
\hline & $\mathrm{C}-5$ & M2-21,M2-22 \\
\hline & C-6 & M2-15,M2-23 \\
\hline & $\mathrm{C}-7$ & M2-17 \\
\hline & $\mathrm{C}-8$ & M2-3 \\
\hline & C-9 & M2-8 \\
\hline & $\mathrm{C}-10$ & M2-17 \\
\hline & $\mathrm{C}-11$ & M2-7 \\
\hline & $\mathrm{C}-12$ & M2-18 \\
\hline \multirow{4}{*}{$\begin{array}{l}\text { Quality } \\
\text { Constraints }\end{array}$} & $\mathrm{C}-2$ & M2-13,M2-14 \\
\hline & $\mathrm{C}-3$ & M2-11,M2-12 \\
\hline & $\mathrm{C}-13$ & M2-16 \\
\hline & C-14 & M2-24 \\
\hline \multirow{3}{*}{$\begin{array}{l}\text { Variable } \\
\text { Bounds }\end{array}$} & $\mathrm{C}-15$ & Not explicitly specified \\
\hline & $\mathrm{C}-16$ & Not explicitly specified \\
\hline & $\mathrm{C}-17$ & Not explicitly specified \\
\hline
\end{tabular}

